\title{
Exploring the three flavor effects with future superbeams using liquid argon detectors
}

\author{
Sanjib Kumar Agarwalla, ${ }^{a}$ Suprabh Prakash $^{b}$ and S. Uma Sankar ${ }^{b}$ \\ ${ }^{a}$ Institute of Physics, Sachivalaya Marg, Sainik School Post, \\ Bhubaneswar 751005, India \\ ${ }^{b}$ Department of Physics, Indian Institute of Technology Bombay, \\ Mumbai 4000\%6, India \\ E-mail: sanjib@iopb.res.in, suprabh@phy.iitb.ac.in, uma@phy.iitb.ac.in
}

ABSTRACT: Recent measurement of a moderately large value of $\theta_{13}$ signifies an important breakthrough in establishing the standard three flavor oscillation picture of neutrinos. It has provided an opportunity to explore the sub-dominant three flavor effects in present and future long-baseline experiments. In this paper, we perform a comparative study of the physics reach of two future superbeam facilities, LBNE and LBNO in their first phases of run, to resolve the issues of neutrino mass hierarchy, octant of $\theta_{23}$, and leptonic $\mathrm{CP}$ violation. We also find that the sensitivity of these future facilities can be improved significantly by adding the projected data from $\mathrm{T} 2 \mathrm{~K}$ and $\mathrm{NO} \nu \mathrm{A}$. Stand-alone LBNO setup with a $10 \mathrm{kt}$ detector has a mass hierarchy discovery reach of more than $7 \sigma$, for the lowest allowed value of $\sin ^{2} \theta_{23}$ (true) $=0.34$. This result is valid for any choice of true $\delta_{\mathrm{CP}}$ and hierarchy. LBNE10, in combination with $\mathrm{T} 2 \mathrm{~K}$ and $\mathrm{NO} \nu \mathrm{A}$, can achieve $3 \sigma$ hierarchy discrimination for any choice of $\delta_{\mathrm{CP}}, \sin ^{2} \theta_{23}$, and hierarchy. The same combination can provide a $3 \sigma$ octant resolution for $\sin ^{2} \theta_{23}$ (true) $\leq 0.44$ or for $\sin ^{2} \theta_{23}$ (true) $\geq 0.58$ for all values of $\delta_{\mathrm{CP}}$ (true). LBNO can give similar results with $10 \mathrm{kt}$ detector mass. In their first phases, both LBNE10 and LBNO with $20 \mathrm{kt}$ detector can establish leptonic CP violation for around $50 \%$ values of true $\delta_{\mathrm{CP}}$ at $2 \sigma$ confidence level. In case of LBNE10, CP coverage at $3 \sigma$ can be enhanced from $3 \%$ to $43 \%$ by combining $\mathrm{T} 2 \mathrm{~K}$ and $\mathrm{NO} \nu \mathrm{A}$ data, assuming $\sin ^{2} \theta_{23}$ (true) $=0.5$. For LBNO setup, CP violation discovery at $3 \sigma$ is possible for $46 \%$ values of true $\delta_{\mathrm{CP}}$ if we add the data from $\mathrm{T} 2 \mathrm{~K}$ and $\mathrm{NO} \nu \mathrm{A}$.

KeYwords: Neutrino Physics, CP violation

ARXIV EPRINT: 1304.3251 


\section{Contents}

1 Introduction and motivation 1

2 Platinum channel: test bed for three flavor effects 3

3 Experimental specifications $\quad 4$

3.1 Current generation: $\mathrm{T} 2 \mathrm{~K}$ and $\mathrm{NO} \nu \mathrm{A}$

3.2 Future generation: LBNE and LBNO 5

4 Physics with bi-events plot $\quad 6$

5 Our findings $\quad 7$

$\begin{array}{lll}5.1 & \text { Discovery reach for neutrino mass hierarchy } & 7\end{array}$

$\begin{array}{ll}5.2 \text { Discovery reach for } \theta_{23} \text { octant } & 10\end{array}$

$\begin{array}{ll}\text { 5.3 Discovery reach for leptonic CP violation } & 13\end{array}$

6 Concluding remarks $\quad 15$

A Resolution of octant as a function of true $\theta_{23}$ for IH(true) 17

B CP violation discovery as a function of true $\delta_{\mathrm{CP}}$ for IH(true) 18

\section{Introduction and motivation}

The discovery of neutrino oscillations over the past decade provides firm evidence for new physics. Recently, the unknown 1-3 lepton mixing angle has been measured quite precisely by the reactor experiments [1-4]. They have found a moderately large value, not too far from its previous upper bound. This represents a significant milestone towards addressing the remaining fundamental questions, in particular determining the neutrino mass hierarchy and searching for $\mathrm{CP}$ violation in the neutrino sector. Another recent and crucial development is the indication of non-maximal 2-3 mixing by the MINOS accelerator experiment $[5,6]$, leading to the problem of determining the correct octant of $\theta_{23}$. It is possible to resolve all the above three issues by the observation of $\nu_{e}$ appearance via $\nu_{\mu} \rightarrow \nu_{e}$ oscillations. The determination of $\mathrm{CP}$ violation in particular requires the full interplay of three flavor effects in neutrino oscillations.

Oscillation data are insensitive to the lowest neutrino mass. However, it can be measured in tritium beta decay processes [7], neutrinoless double beta decay experiments [8], and from the contribution of neutrinos to the energy density of the universe [9]. Very recent data from the Planck experiment in combination with the WMAP polarization and baryon acoustic oscillation measurements have set an upper bound on the sum of all the neutrino 
mass eigenvalues of $\sum m_{i} \leq 0.23 \mathrm{eV}$ at $95 \%$ C.L. [10]. But, oscillation experiments are capable of measuring the two independent mass-squared differences: $\Delta m_{21}^{2}=m_{2}^{2}-m_{1}^{2}$ and $\Delta m_{31}^{2}=m_{3}^{2}-m_{1}^{2} . \Delta m_{21}^{2}$ is required to be positive by the solar neutrino data but at present $\Delta m_{31}^{2}$ can be either positive or negative. Hence, two patterns of neutrino masses are possible: $m_{3}>m_{2}>m_{1}$, called normal hierarchy $(\mathrm{NH})$ where $\Delta m_{31}^{2}$ is positive and $m_{2}>m_{1}>m_{3}$, called inverted hierarchy (IH) where $\Delta m_{31}^{2}$ is negative. Leptonic CP violation can be established if CP violating phase $\delta_{\mathrm{CP}}$ in the mixing matrix, differs from both 0 and $180^{\circ}$. Recent global fit of neutrino oscillation data $[11,12]$ indicates a preference for negative values of $\delta_{\mathrm{CP}}$ at $2 \sigma$ C.L., ruling out the possibility of $\delta_{\mathrm{CP}}$ being $90^{\circ}$ at around $2.5 \sigma$. This feature mainly arises due to the present tension between the reactor and T2K data. In [13], combining all the oscillation data, the authors claim to find a hint for non-zero CP violation $\left(\sin \delta_{\mathrm{CP}}<0\right.$ at $\sim 90 \%$ C.L.) assuming $\mathrm{NH}$. However, in this paper, we take a conservative approach and vary $\delta_{\mathrm{CP}}$ in the full range of $\left[-180^{\circ}, 180^{\circ}\right]$. Regarding $\theta_{23}$, combined analysis of all the neutrino oscillation data available disfavors the maximal mixing solution for $\theta_{23}$ at $1.4 \sigma$ confidence level $[11,12]$. It suggests the deviation of $\theta_{23}$ from maximal mixing $(\mathrm{MM})$ i.e. $\left(0.5-\sin ^{2} \theta_{23}\right) \neq 0$. This raises an additional question: "whether $\theta_{23}$ lies in the lower octant (LO: $\theta_{23}<45^{\circ}$ ) or higher octant (HO: $\theta_{23}>45^{\circ}$ )?" [14].

Settling the issue of neutrino mass hierarchy is crucial to determine the structure of neutrino mass matrix. This structure will provide the fundamental input needed to develop the theory of neutrino masses and mixing [15]. Neutrino mass hierarchy is also a key parameter for neutrinoless double beta decay searches probing the Majorana nature of neutrinos [16]. Another fundamental issue that needs to be addressed in long-baseline experiments is to establish leptonic $\mathrm{CP}$ violation and measure $\delta_{\mathrm{CP}}$. It is conjectured that the matter anti-matter asymmetry of the universe arose via leptogenesis [17], which requires the existence of $\mathrm{CP}$ violation in the leptonic sector. The phases leading to the $\mathrm{CP}$ violation in neutrino oscillations and in leptogenesis are in general quite different. But, the discovery of $\mathrm{CP}$ violation in the leptonic sector will be a very exciting discovery and will make leptogenesis more attractive. It will also be a very valuable input for constructing appropriate neutrino mass matrices. A number of innovative ideas, such as $\mu \leftrightarrow \tau$ symmetry [18], $A_{4}$ flavor symmetry [19], quark-lepton complementarity [20], and neutrino mixing anarchy $[21,22]$ have been invoked to explain the observed pattern of one small and two large mixing angles in the neutrino sector. Measurements of the precise values of $\theta_{13}$ and $\theta_{23}$ will reveal the pattern of deviations from these symmetries and will lead to a better understanding of neutrino masses and mixing. In particular, the resolution of $\theta_{23}$ octant will severely constrain the patterns of symmetry breaking. With the recent discovery of moderately large value of $\theta_{13}$, these three fundamental measurements fall within our reach.

The combined data from the current $\nu_{e}$ appearance experiments, T2K $[23,24]$ and $\mathrm{NO} \nu \mathrm{A}[25-27]$, can provide a hint at $90 \%$ confidence level for neutrino mass ordering [28, 29] and at $95 \%$ confidence level for octant of $\theta_{23}$ [30, 31]. They can determine these quantities at $>99 \%$ C.L. only for a very small range of favorable values of $\delta_{\mathrm{CP}}$. Discovery of leptonic CP violation is possible at $95 \%$ C.L. only for values of $\delta_{\mathrm{CP}}$ close to $\pm 90^{\circ}$, i.e. where $\mathrm{CP}$ violation is maximum [28, 29]. Hence, future facilities consisting of intense, high power wide-band beams and large smart detectors are mandatory to cover the entire 
parameter space at a high confidence level. In this paper, we explore the capabilities of future superbeam experiments with liquid argon detectors, LBNE [32-36] and LBNO [3741] towards resolving these unknowns. We first present the stand-alone performances of these setups in their first phases. Then we examine how the addition of projected data from $\mathrm{T} 2 \mathrm{~K}$ and $\mathrm{NO} \nu \mathrm{A}$, can improve the sensitivity of these future facilities. We also study in detail how these sensitivities change as the true value of $\sin ^{2} \theta_{23}$ varies in its allowed $3 \sigma$ range of 0.34 to 0.67 .

We start with a brief discussion of $\nu_{\mu} \rightarrow \nu_{e}$ oscillation channel in section 2. In section 3, we describe the important features of the experimental setups under consideration. Next, we introduce the concept of bi-events plots $\left(\nu_{e}\right.$ vs. $\bar{\nu}_{e}$ appearance events) to explain the underlying physics in section 4 . In section 5 , we present our results. Finally, we summarize and draw our conclusions in section 6.

\section{Platinum channel: test bed for three flavor effects}

A study of $\nu_{\mu} \rightarrow \nu_{e}$ and $\bar{\nu}_{\mu} \rightarrow \bar{\nu}_{e}$ oscillations at long-baseline superbeam experiments is the simplest way to probe three flavor effects, including sub-leading ones. Such a study is capable of achieving all the three objectives mentioned in section 1. An approximate analytic expression for the oscillation probability, $P_{\mu e}$, in matter [42-44], is given by

$$
\begin{aligned}
& P_{\mu e} \simeq \underbrace{\sin ^{2} \theta_{23} \sin ^{2} 2 \theta_{13} \frac{\sin ^{2}[(1-\hat{A}) \Delta]}{(1-\hat{A})^{2}}}_{C_{0}}+\underbrace{\alpha^{2} \cos ^{2} \theta_{23} \sin ^{2} 2 \theta_{12} \frac{\sin ^{2}(\hat{A} \Delta)}{\hat{A}^{2}}}_{C_{1}} \\
& \underbrace{\alpha \sin 2 \theta_{13} \cos \theta_{13} \sin 2 \theta_{12} \sin 2 \theta_{23} \sin (\Delta) \frac{\sin (\hat{A} \Delta)}{\hat{A}} \frac{\sin [(1-\hat{A}) \Delta]}{(1-\hat{A})}}_{C_{-}} \sin \delta_{\mathrm{CP}} \\
& +\underbrace{\alpha \sin 2 \theta_{13} \cos \theta_{13} \sin 2 \theta_{12} \sin 2 \theta_{23} \cos (\Delta) \frac{\sin (\hat{A} \Delta)}{\hat{A}} \frac{\sin [(1-\hat{A}) \Delta]}{(1-\hat{A})}}_{C_{+}} \cos \delta_{\mathrm{CP}}
\end{aligned}
$$

where

$$
\Delta \equiv \frac{\Delta m_{31}^{2} L}{4 E}, \quad \hat{A} \equiv \frac{A}{\Delta m_{31}^{2}}, \quad A= \pm 2 \sqrt{2} G_{F} N_{e} E
$$

Equation 2.1 has been derived under the constant matter density approximation, keeping terms only up to second order in the small quantities $\theta_{13}$ and $\alpha \equiv \Delta m_{21}^{2} / \Delta m_{31}^{2}[45-47]$. Here, $A$ is the matter potential, expressed in terms of the electron density, $N_{e}$, and the (anti-)neutrino energy $E$. It is positive for neutrinos and negative for anti-neutrinos. For anti-neutrinos, the term proportional to $\sin \delta_{\mathrm{CP}}$ has the opposite sign. So far, it was possible to analyze the data from each oscillation experiment using an appropriate, effective two flavor oscillation approach because of the smallness of the mixing angle $\sin 2 \theta_{13} \simeq 0.3$ and the ratio $\alpha \simeq 0.03$. This method has been quite successful in measuring the solar and 
atmospheric neutrino parameters. The next step must involve probing the full three flavor effects, including the sub-leading ones proportional to $\alpha$. This task will be undertaken, for the first time, by the current generation experiments $\mathrm{T} 2 \mathrm{~K}$ and $\mathrm{NO} \nu \mathrm{A}$.

In this paper, we consider two future long-baseline superbeam experiments with large matter effect. The matter effect increases $P\left(\nu_{\mu} \rightarrow \nu_{e}\right)$ oscillation probability for $\mathrm{NH}$ and decreases it for IH. For anti-neutrinos the situation is reversed. It can be seen from equation (2.1) that the dominant term $\left(C_{0}\right)$ is driven by matter modified $\Delta m_{31}^{2}$ and is proportional to $\sin ^{2} \theta_{23} \sin ^{2} 2 \theta_{13}$ but the sub-dominant $\delta_{\mathrm{CP}}$ dependent terms $\left(C_{-} \& C_{+}\right)$are suppressed by $\alpha$. Since the hierarchy and $\delta_{\mathrm{CP}}$ are both unknown, the interplay of the terms $C_{0}, C_{-}$, and $C_{+}$in equation (2.1) gives rise to hierarchy- $\delta_{\mathrm{CP}}$ degeneracy [48]. If the matter effects are large enough, this degeneracy can be broken completely. This is not the case for $\mathrm{T} 2 \mathrm{~K}$ and $\mathrm{NO} \nu \mathrm{A}$, because of which their sensitivity to hierarchy is modest for about half the $\delta_{\mathrm{CP}}$ range. There is a similar octant- $\delta_{\mathrm{CP}}$ degeneracy also, which limits our ability to determine the correct octant of $\theta_{23}$ [14]. This problem can be solved by having substantial data in both $\nu$ and $\bar{\nu}$ channels [30, 49]. Both the future facilities, LBNE (baseline of 1300 $\mathrm{km}$ ) and LBNO (baseline of $2290 \mathrm{~km}$ ) will operate at multi-GeV energies with very longbaselines. This will lead to a large enough matter effect which breaks the hierarchy- $\delta_{\mathrm{CP}}$ degeneracy completely. They are also scheduled to have equal $\nu$ and $\bar{\nu}$ runs, and can resolve the octant $-\delta_{\mathrm{CP}}$ degeneracy effectively. These experiments are planning to use liquid argon time projection chambers (LArTPCs) which have excellent kinematic reconstruction capability for all the observed particles. This feature helps in rejecting almost all of the large neutral current background.

\section{Experimental specifications}

In this section, we briefly describe the key experimental features of the current (off-axis) and future (on-axis) generation long-baseline superbeam experiments that we use in our simulation.

\subsection{Current generation: $\mathrm{T} 2 \mathrm{~K}$ and $\mathrm{NO} \nu \mathrm{A}$}

In Japan, the Tokai-to-Kamioka (T2K) experiment [23, 24] started taking data in 2010. The NO $\nu \mathrm{A}$ experiment [25-27] in the United States is now under construction and will start taking data near the end of this year. The main goal of these experiments is to detect the electron neutrino appearance events in a $\nu_{\mu}$ beam using the classic off-axis beam technique [50] that delivers a beam with a narrow peak in the energy spectrum. The position of this peak is tuned to be close to the expected oscillation maximum. In our study, we have explored the improvement in the physics capabilities of LBNE and LBNO in their first phases, due to the addition of the projected data from $\mathrm{T} 2 \mathrm{~K}$ and $\mathrm{NO} \nu \mathrm{A}$ experiments.

In the T2K experiment, a $2.5^{\circ}$ off-axis $\nu_{\mu}$ beam from J-PARC is observed in the SuperKamiokande detector (fiducial volume $22.5 \mathrm{kt}$ ) at Kamioka, at a distance of $295 \mathrm{~km}$ [23]. The neutrino flux peaks sharply at the first oscillation maximum of $0.6 \mathrm{GeV}$. For mass hierarchy and $\mathrm{CP}$ violation studies, we consider 5 years of neutrino run with a beam power 
of $0.75 \mathrm{MW}$ as officially announced. ${ }^{1}$ Recently, it has been shown in reference [30] that equal runs in neutrino and anti-neutrino modes in $\mathrm{T} 2 \mathrm{~K}$ experiments are vital to settle the octant ambiguity of $\theta_{23}$ for all values of $\delta_{\mathrm{CP}}$. Therefore, we assume equal neutrino and anti-neutrino runs of 2.5 years each for the T2K while exploring the octant sensitivity. The signal efficiency in T2K is around $87 \%$. In our simulation, the background information and other details for T2K experiment are taken from [28, 52].

In the $\mathrm{NO} \nu \mathrm{A}$ experiment, the NuMI beam will be sent towards a $14 \mathrm{kt}$ totally active scintillator detector (TASD) placed at a distance of $810 \mathrm{~km}$ from Fermilab, at a location which is $0.8^{\circ}$ off-axis from the beam. Due to the off-axis location, the flux is sharply peaked around $2 \mathrm{GeV}$, again close to the first oscillation maximum in $P\left(\nu_{\mu} \rightarrow \nu_{e}\right)$ channel. The experiment is scheduled to have three years run in neutrino mode first and then later, three years run in anti-neutrino mode. The NuMI beam power is $0.7 \mathrm{MW}$, which corresponds to $6 \times 10^{20}$ p.o.t. per year. See, reference [27] for details. After the discovery of moderately large value of $\theta_{13}, \mathrm{NO} \nu \mathrm{A}$ has reoptimized its event selection criteria. A few cuts have been relaxed to allow more events in both signal and background. Additional neutral current backgrounds are reconstructed at lower energies and can be rejected by a kinematical cut. In our simulation, we use all these new features, the details of which are given in $[29,53]$.

\subsection{Future generation: LBNE and LBNO}

The Long-Baseline Neutrino Experiment (LBNE) [35, 36] is one of the major components of Fermilab's intensity frontier program. In its first phase (LBNE10), it will have a new, high intensity, on-axis neutrino beam directed towards a $10 \mathrm{kt}$ LArTPC located at Homestake with a baseline of $1300 \mathrm{~km}$. This facility is designed for initial operation at a proton beam power of $708 \mathrm{~kW}$, with proton energy of $120 \mathrm{GeV}$ that will deliver $6 \times 10^{20}$ p.o.t. in 230 days per calendar year. In our simulation, we have used the latest fluxes being considered by the collaboration, which have been estimated assuming the smaller decay pipe and the lower horn current compared to the previous studies [54]. We have assumed five years each of $\nu$ and $\bar{\nu}$ runs. The detector characteristics have been taken from table 1 of [55]. To have the LArTPC cross-sections, we have scaled the inclusive charged current (CC) cross sections of water by $1.06(0.94)$ for the $\nu(\bar{\nu})$ case $[56,57]$.

The Long-Baseline Neutrino Oscillation Experiment (LBNO) [41] plans to use an experimental set-up where neutrinos produced in a conventional wide-band beam facility at CERN would be observed in a proposed $20 \mathrm{kt}$ (in its first phase) LArTPC housed at the Pyhäsalmi mine in Finland, at a distance of $2290 \mathrm{~km}$. The fluxes have been computed [58] assuming an exposure of $1.5 \times 10^{20}$ p.o.t. in 200 days per calendar year from the SPS accelerator at $400 \mathrm{GeV}$ with a beam power of $750 \mathrm{~kW}$. For LBNO also, we consider five years each of $\nu$ and $\bar{\nu}$ runs. We assume the same detector properties as that of LBNE10. In our calculations, we also consider a LBNO configuration reducing the detector mass to

\footnotetext{
${ }^{1}$ Currently, T2K is operating at a beam power of $220 \mathrm{~kW}$ and has delivered a total $6.63 \times 10^{20}$ protons on target (p.o.t.) [51]. It is expected that the planned upgrade of the J-PARC Main Ring will be completed by 2018. It will enable $\mathrm{T} 2 \mathrm{~K}$ to deliver the full planned statistics of $78 \times 10^{20}$ p.o.t. by the end of 2020 which is equivalent to a 5 years run at $0.75 \mathrm{MW}$. The future experiments, LBNE or LBNO, are likely start only after that.
} 


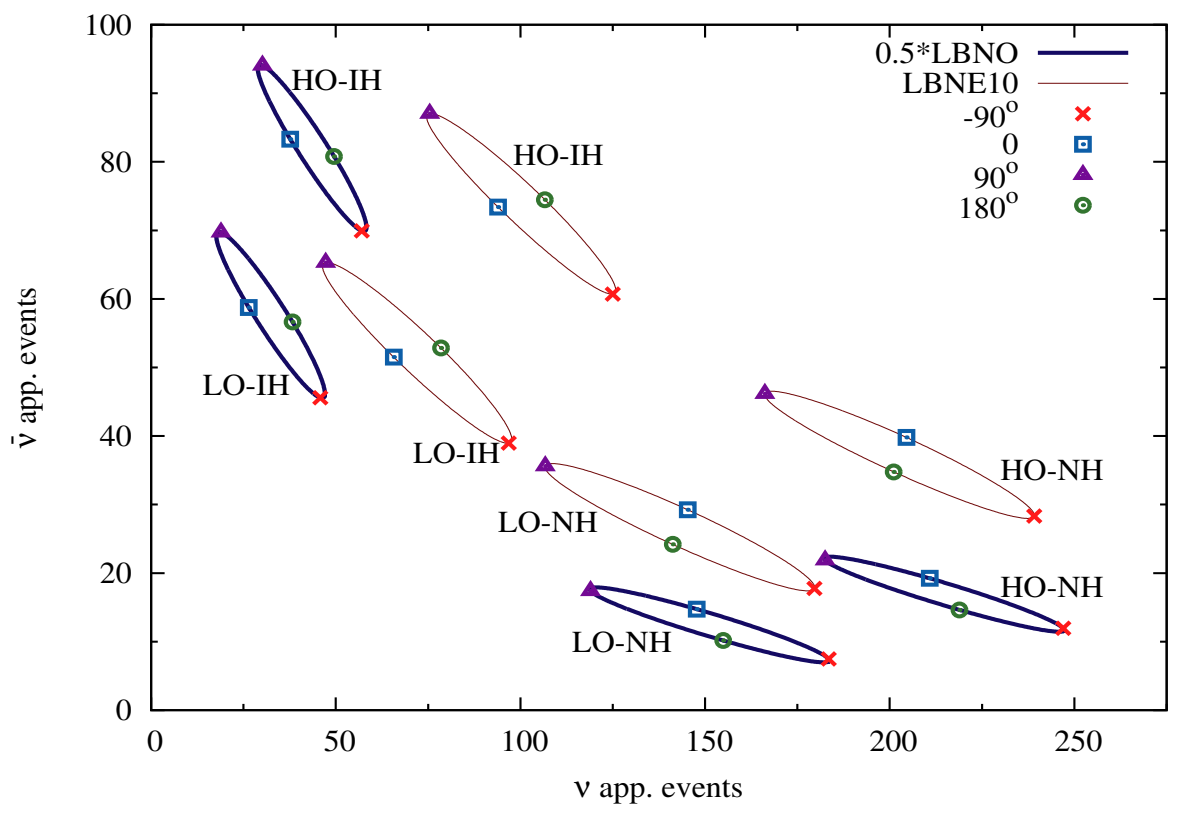

Figure 1. Bi-events $\left(\nu_{e}\right.$ and $\bar{\nu}_{e}$ appearance) plot for the four possible octant-hierarchy combinations and all possible $\delta_{\mathrm{CP}}$ values. The experiments considered are LBNE10 and $0.5^{*} \mathrm{LBNO}$. Here $\sin ^{2} 2 \theta_{13}=0.089$. For LO (HO), $\sin ^{2} \theta_{23}=0.41(0.59)$.

$10 \mathrm{kt}$ which we denote as $0.5^{*} \mathrm{LBNO}$. The exposure for this setup will be quite similar to LBNE10 which will enable us to perform a comparative study between these two setups at the same footing. The results presented in this paper are obtained using the GLoBES software [59, 60].

\section{Physics with bi-events plot}

In this section, we attempt to understand the physics capabilities of $0.5^{*} \mathrm{LBNO}$ and LBNE10 setups with the help of bi-events plot. This kind of plot is quite useful to get a qualitative estimate of the physics sensitivity before performing a full $\Delta \chi^{2}$ calculation. In figure 1 , we have plotted $\nu_{e}$ vs. $\bar{\nu}_{e}$ appearance events, for $0.5^{*} \mathrm{LBNO}$ and LBNE10 for the four possible combinations of hierarchy and octant. Since $\delta_{\mathrm{CP}}$ is unknown, events are generated for the full range $\left[-180^{\circ}, 180^{\circ}\right]$, leading to the ellipses. The event rates are calculated using the following oscillation parameters: $\Delta m_{21}^{2}=7.5 \times 10^{-5} \mathrm{eV}^{2}, \sin ^{2} \theta_{12}=0.3$ [12], $\Delta \mathrm{m}_{\text {eff }}^{2}= \pm 2.4 \times 10^{-3} \mathrm{eV}^{2}[6]$, and $\sin ^{2} 2 \theta_{13}=0.089$ [2]. $\Delta \mathrm{m}_{\text {eff }}^{2}$ is the effective mass-squared difference measured using the $\nu_{\mu}$ survival probability and is a linear combination of $\Delta m_{31}^{2}$ and $\Delta m_{21}^{2}$. The value of $\Delta m_{31}^{2}$ is derived from $\Delta \mathrm{m}_{\text {eff }}^{2}$ using the relation given in [61, 62]. This relation leads to different magnitudes of $\Delta m_{31}^{2}$ for NH and for IH. For $\sin ^{2} \theta_{23}$, we choose the two degenerate best-fit values of the global fit [12]: 0.41 in the lower octant (LO) and 0.59 in the higher octant (HO). Note that, here we have plotted the total number of events, whereas the actual analysis will be done based on the spectral information. Nevertheless, the contours in this figure contain very important information regarding the 
physics capabilities of the experiments. An experiment can determine both the hierarchy and the octant, if every point on a given ellipse is well separated from every point on each of the other three ellipses. The larger the separation, the better is the confidence level with which the above parameters can be determined.

One can see from figure 1 that for $0.5^{*} \mathrm{LBNO}$, the two (LO/HO)-IH ellipses are well separated from the two (LO/HO)-NH ellipses, in number of $\nu_{e}$ events. Hence, $0.5^{*} \mathrm{LBNO}$ has excellent hierarchy determination capability with just $\nu$ data. However, $\nu$ data alone will not be sufficient to determine the octant in case of $\mathrm{IH}$, because various points on (LO/HO)-IH ellipses have the same number of $\nu_{e}$ events. Likewise, only $\bar{\nu}$ data cannot determine the octant in case of $\mathrm{NH}$. Therefore, balanced $\nu$ and $\bar{\nu}$ data are mandatory to make an effective distinction between ( $\mathrm{LO} / \mathrm{HO})-\mathrm{IH}$ ellipses and also between ( $\mathrm{LO} / \mathrm{HO})-\mathrm{NH}$ ellipses. Figure 1 also depicts that the asymmetries between the neutrino and anti-neutrino appearance events are largest for the combinations: $\left(\mathrm{NH}, \delta_{\mathrm{CP}}=-90^{\circ}\right)$ and $\left(\mathrm{IH}, \delta_{\mathrm{CP}}=90^{\circ}\right)$.

For LBNE10, $\nu$ data alone can not determine hierarchy because various points on LO$\mathrm{NH}$ and HO-IH ellipses have the same number of $\nu_{e}$ events (see figure 1). Thus, $\bar{\nu}$ data is also needed. Even with $\bar{\nu}$ data, hierarchy determination can be difficult to achieve, if nature chooses LO and one of the two worst case combinations of hierarchy and $\delta_{\mathrm{CP}}$ which are $(\mathrm{NH}$, $\left.90^{\circ}\right)$ or $\left(\mathrm{IH},-90^{\circ}\right)$. In such a situation, the $\nu_{e}$ and $\bar{\nu}_{e}$ events are rather close to each other and it will be very difficult for LBNE10 to reject the wrong combination. Regarding octant determination, the capability of LBNE10 is very similar to that of $0.5^{*} \mathrm{LBNO}$ because the separations between the ellipses, belonging to LO and HO are very similar for these two experiments.

\section{Our findings}

Measurement of mass hierarchy and octant should be considered as a prerequisite for the discovery of leptonic CP violation. Now, it would be quite interesting to study whether the expected appearance data from the first phases of LBNE and LBNO experiments can resolve the issues of neutrino mass hierarchy and octant of $\theta_{23}$ at $3 \sigma$ to $5 \sigma$ confidence level before they start probing the parameter space for leptonic CP violation. In this section, we address these issues in detail. We present the results for LBNE10 (10 kt), 0.5*LBNO (10 $\mathrm{kt})$, and LBNO (20 kt) setups. We also study the improvement in their physics reach when the projected data from current generation experiments $\mathrm{T} 2 \mathrm{~K}$ and $\mathrm{NO} \nu \mathrm{A}$, is added. The impact of $\mathrm{T} 2 \mathrm{~K}$ and $\mathrm{NO} \nu \mathrm{A}$ measurements on the performance of LBNE setup to determine the mass hierarchy and discover leptonic CP violation has been discussed recently in [63].

\subsection{Discovery reach for neutrino mass hierarchy}

We first focus on the discovery potential of future facilities to exclude the wrong hierarchy. It can be seen from equation (2.2) that the first term $\left(C_{0}\right)$ dominates for large $\theta_{13}$ and it is the leading term in platinum channel. This term contains the largest Earth matter effect which can therefore be used to unravel the sign of $\Delta m_{31}^{2}$. This term is also proportional to $\sin ^{2} \theta_{23}$ and therefore is quite sensitive to the choice of $\theta_{23}$ value. If we vary $\sin ^{2} \theta_{23}$ in its $3 \sigma$ allowed range of 0.34 to 0.67 , then for LBNE10, the signal event rates in $\nu_{e}$ appearance 


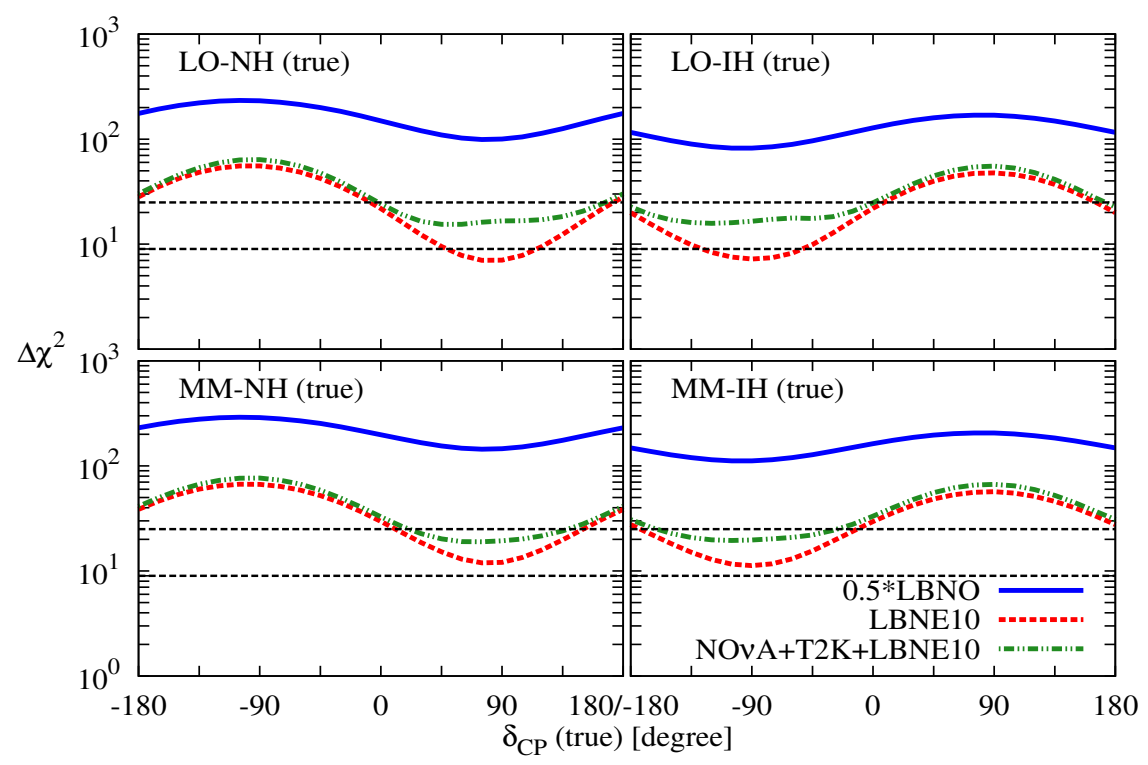

Figure 2. Discovery reach for mass hierarchy as a function of true $\delta_{\mathrm{CP}}$ for $0.5^{*} \mathrm{LBNO}$, LBNE10, and LBNE10 combining the projected data from $\mathrm{T} 2 \mathrm{~K}$ and $\mathrm{NO} \nu \mathrm{A}$ (see section 3). Results are shown for four possible true $\theta_{23}$-hierarchy combinations. For LO (MM), $\sin ^{2} \theta_{23}$ (true) $=0.41(0.5)$. Here $\sin ^{2} 2 \theta_{13}($ true $)=0.089$.

channel will increase from 122 to 231 (assuming $\mathrm{NH}$ and $\delta_{\mathrm{CP}}=0^{\circ}$ ), an almost $\sim 90 \%$ enhancement in the statistics. For LBNO setup with $20 \mathrm{kt}$ detector size, these numbers will change from 247 to 478 showing an almost $\sim 94 \%$ increase in the event numbers. $\Delta \chi^{2}$ is calculated for a given true combination of $\theta_{23}$-hierarchy, assuming the opposite hierarchy to be the test hierarchy. In the fit, we marginalize over test $\sin ^{2} \theta_{23}$ in its $3 \sigma$ range. $\Delta \mathrm{m}_{\text {eff }}^{2}$ and $\sin ^{2} 2 \theta_{13}$ are marginalized in their $2 \sigma$ ranges. We consider $5 \%$ uncertainty in the matter density, $\rho$. Priors were added for $\rho(\sigma=5 \%), \Delta \mathrm{m}_{\text {eff }}^{2}(\sigma=4 \%)$, and $\sin ^{2} 2 \theta_{13}(\sigma=5 \%$, as expected by the end of Daya Bay's run [64]). $\Delta \chi^{2}$ is also marginalized over the uncorrelated systematic uncertainties ( $5 \%$ on signal and $5 \%$ on background) in the set-ups, so as to obtain a $\Delta \chi_{\min }^{2}$ for every $\delta_{\mathrm{CP}}$ (true). Our assumptions of $5 \%$ systematic uncertainties for signal and background are somewhat optimistic. Such assumptions are made by the collaborations [36, 41]. These uncertainties mainly stem from the cross-section, especially for the quasi-elastic $(\mathrm{QE})$ process which dominates at lower energies $(E \leq 1 \mathrm{GeV})$. The peak energies of these future experiments are reasonably high where cross-sections are measured to a better precision. Also, the neutrino scattering experiment, MINERvA [65] is certainly going to improve our knowledge on these cross-sections. Hence, our optimistic assumptions on the systematic uncertainties are not unreasonable. Also, in [66], it is demonstrated that both these future experiments (especially in their first phases) are statistically dominated. For these experiments, near detectors are not mandatory since the combined fit of appearance and disappearance measurements can almost reduce the impact of systematics in a three flavor framework [66]. 
First, we consider two true values of $\sin ^{2} \theta_{23}$ : 0.41 (best-fit value in LO) and 0.5 $(\mathrm{MM})$ giving us four true combinations of $\theta_{23}$-hierarchy: LO-NH, LO-IH, MM-NH and MM-IH. The hierarchy reach would suffer the most if $\sin ^{2} \theta_{23}$ (true) belongs to LO, hence we show the results for the best-fit value in LO. Here, we would like to mention that if we take $\sin ^{2} \theta_{23}$ (true) to be the best-fit value in $\mathrm{HO}$, then the discovery reaches of these experiments will be better than that for the case of MM. We elaborate on this point at the end of this section. Figure 2 depicts the discovery reach for hierarchy as a function of $\delta_{\mathrm{CP}}$ (true). We see that even $0.5^{*} \mathrm{LBNO}$ has $\gtrsim 10 \sigma^{2}$ hierarchy discovery potential for all values of $\delta_{\mathrm{CP}}$ (true) and for all four true $\theta_{23}$-hierarchy combinations. The potential of LBNO, of course, is even better. Also, it was demonstrated earlier that baselines around $L$ $\sim 2500 \mathrm{~km}$ have excellent hierarchy discovery reach [68-70]. For LBNE10, a $5 \sigma$ discovery of hierarchy is possible for only $\sim 50 \%$ of the $\delta_{\mathrm{CP}}$ (true), irrespective of these four true $\theta_{23}$-hierarchy combinations. For the unfavorable hierarchy- $\delta_{\mathrm{CP}}$ combinations [71], i.e. NH with $\delta_{\mathrm{CP}}$ in the upper half plane or IH with $\delta_{\mathrm{CP}}$ in the lower half plane, the performance of LBNE10 suffers. In particular, for LO and the worst case combinations [(NH, $\left.90^{\circ}\right)$ and $\left(\mathrm{IH},-90^{\circ}\right)$ ], LBNE10 will not be able to provide even a $3 \sigma$ hierarchy discrimination. This suggests that additional data is needed for LBNE10 to have such a capability. In such a scenario, the projected data from $\mathrm{T} 2 \mathrm{~K}$ and $\mathrm{NO} \nu \mathrm{A}$ can come to the rescue. Adding data from $\mathrm{T} 2 \mathrm{~K}$ ( 5 years of neutrino run) and $\mathrm{NO} \nu \mathrm{A}$ ( 3 years of $\nu$ run and 3 years of $\bar{\nu}$ run) helps LBNE10 setup to achieve more than $3 \sigma$ discovery reach for mass hierarchy irrespective of the true choices of hierarchy and $\delta_{\mathrm{CP}}$ (see upper panels of figure 2), even if $\theta_{23}$ is in the lower octant.

Now, we ask the question, by how much does the sensitivity deteriorate if $\sin ^{2} \theta_{23}$ (true) turns out to be 0.34 in nature, which is its minimum value allowed in the $3 \sigma$ range? We have checked that even in this case, LBNO setup with $20 \mathrm{kt}$ detector mass can give $\Delta \chi_{\min }^{2} \gtrsim 100$ irrespective of the true choices of hierarchy, and $\delta_{\mathrm{CP}}$. From figure 3 , it can be seen that $0.5^{*} \mathrm{LBNO}$ can resolve the issue of mass hierarchy at more than $7 \sigma$ confidence level for $\sin ^{2} \theta_{23}$ (true) $=0.34$ independent of the choices of true hierarchy and $\delta_{\mathrm{CP}}$. The most important message that is conveyed by figure 3 is that with the help of projected T2K and $\mathrm{NO} \nu \mathrm{A}$ data, LBNE10 can still achieve $3 \sigma$ mass hierarchy discovery for any combinations of true hierarchy- $\delta_{\mathrm{CP}}-\sin ^{2} \theta_{23}$. It clearly demonstrates the synergy between the current (off-axis) and future (on-axis) superbeam experiments and also proves that adding data from three different baselines $(295 \mathrm{~km}, 810 \mathrm{~km}$, and $1300 \mathrm{~km})$ with completely different energy spectra is quite useful to kill the clone solutions for the unfavorable choices of the oscillation parameters.

The mass hierarchy discovery potential for all the three set-ups under consideration is remarkable if $\theta_{23}$ happens to lie in HO. For $\sin ^{2} \theta_{23}$ (true) $=0.59$ (the best-fit value in $\mathrm{HO})$, even $0.5^{*} \mathrm{LBNO}$ can have $\Delta \chi_{\min }^{2} \gtrsim 130$ irrespective of the true choices of hierarchy and $\delta_{\mathrm{CP}}$. With this choice of $\sin ^{2} \theta_{23}$ (true), a $5 \sigma$ discovery is not possible with LBNE10 for $\sim 30 \%$ values of true $\delta_{\mathrm{CP}}$ in the upper half plane for $\mathrm{NH}$ true and for $\sim 70 \%$ values of

\footnotetext{
${ }^{2}$ To estimate this, we use the relation $\mathrm{n} \sigma=\sqrt{\Delta \chi_{\min }^{2}}$. It is shown in a recent paper [67] that the above relation is valid in the frequentist method of hypothesis testing.
} 

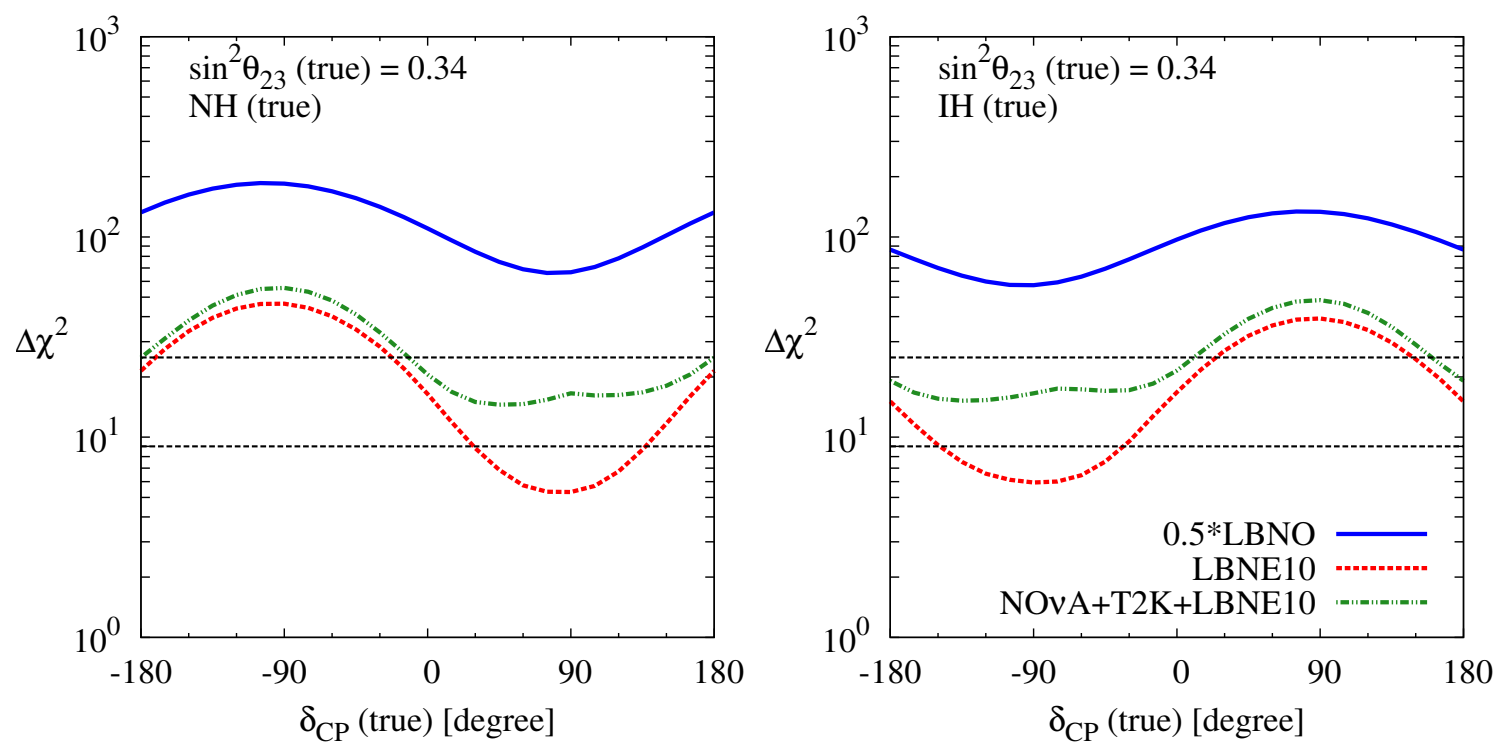

Figure 3. Left panel (right panel) shows the $\Delta \chi^{2}$ for the mass hierarchy discovery as a function of true value of $\delta_{\mathrm{CP}}$ assuming $\mathrm{NH}(\mathrm{IH})$ as true hierarchy. Results are shown for $0.5^{*} \mathrm{LBNO}, \mathrm{LBNE} 10$, and $\mathrm{LBNE} 10+\mathrm{T} 2 \mathrm{~K}+\mathrm{NO} \nu \mathrm{A}$. Here we consider $\sin ^{2} \theta_{23}$ (true) $=0.34$ (the lowest value in its allowed $3 \sigma$ range).

true $\delta_{\mathrm{CP}}$ in the lower half plane for IH true. We have checked that if we add the data from $\mathrm{T} 2 \mathrm{~K}$ and $\mathrm{NO} \nu \mathrm{A}, \mathrm{LBNE} 10$ can again provide $5 \sigma$ discovery for mass hierarchy irrespective of the choices of true hierarchy and $\delta_{\mathrm{CP}}$ with $\sin ^{2} \theta_{23}$ (true) $=0.59$.

We find that our results for hierarchy determination in the case of LBNE10 are matching quite well with the results shown in [36]. Figure 4-13 (top panels) of [36] shows the results for hierarchy where different assumptions on the systematic uncertainties and beam power have been made. We see a $3 \sigma$ to $10 \sigma$ variation in the discovery reach depending on the true $\delta_{\mathrm{CP}}$. In the case of LBNO, our results broadly agree with that in figure 72 of [41] where we see a greater than $5 \sigma$ discovery of hierarchy for any true value of $\delta_{\mathrm{CP}}$. Next we turn our attention to the octant discovery potential of these setups.

\subsection{Discovery reach for $\theta_{23}$ octant}

Here we discuss the discovery reach of future facilities for excluding the wrong octant. We consider the best-fit true values of $\sin ^{2} \theta_{23}=0.41$ (in LO) and 0.59 (in HO) resulting into the following four true combinations of octant and hierarchy: LO-NH, LO-IH, HO-NH, and HO-IH. $\Delta \chi^{2}$ is calculated for each of these four combinations, assuming test $\sin ^{2} \theta_{23}$ values from the other octant. For LO (HO) true, we consider the test $\sin ^{2} \theta_{23}$ range from 0.5 to 0.67 (0.34 to 0.5). Rest of the marginalization procedure (over other oscillation parameters and systematic uncertainties) is the same as that in the case of hierarchy exclusion except with another difference: the final $\Delta \chi^{2}$ is marginalized over both the hierarchies in the fit to obtain $\Delta \chi_{\min }^{2}$.

Figure 4 shows the discovery reach for octant as a function of $\delta_{\mathrm{CP}}($ true). It can be seen that for $(\mathrm{LO} / \mathrm{HO})-\mathrm{IH}$ true, the sensitivities of LBNE10 and $0.5^{*} \mathrm{LBNO}$ are quite similar 


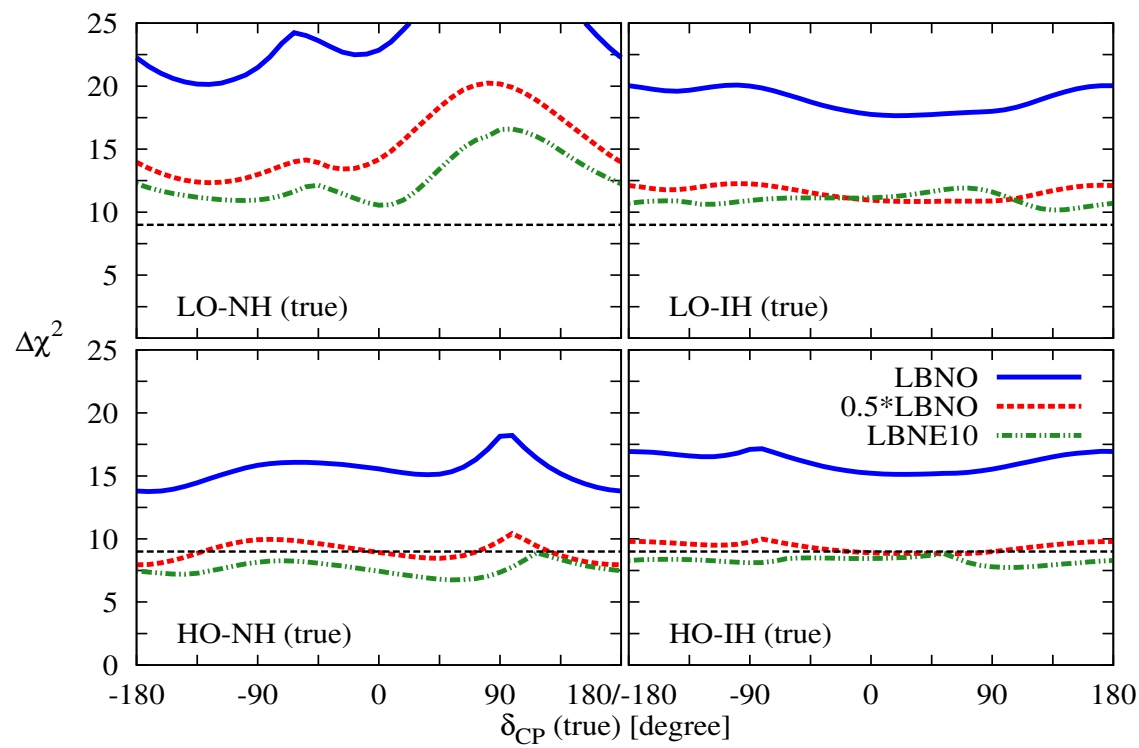

Figure 4. Octant resolving capability as a function of true $\delta_{\mathrm{CP}}$ for LBNO, 0.5*LBNO, and LBNE10. Results are shown for the four possible true octant-hierarchy combinations. For LO $(\mathrm{HO}), \sin ^{2} \theta_{23}($ true $)=0.41(0.59)$. Here $\sin ^{2} 2 \theta_{13}($ true $)=0.089$.

whereas they are somewhat better for $0.5^{*} \mathrm{LBNO}$ if $(\mathrm{LO} / \mathrm{HO})-\mathrm{NH}$ are the true combinations. For LO- $(\mathrm{NH} / \mathrm{IH})$, both LBNE10 and $0.5^{*} \mathrm{LBNO}$ have more than $3 \sigma$ discovery of octant while for $\mathrm{HO}-(\mathrm{NH} / \mathrm{IH})$, the $\Delta \chi_{\min }^{2}$ varies from $\sim 7$ to 11 depending on the true value of $\delta_{\mathrm{CP}}$. However, with full LBNO, we have more than $3.5 \sigma$ discovery of octant for all true octant-hierarchy- $\delta_{\mathrm{CP}}$ combinations. A $5 \sigma$ discovery of octant is possible only for LO-NH true for $\delta_{\mathrm{CP}}($ true $) \in\left(\sim 20^{\circ}\right.$ to $\left.150^{\circ}\right)$.

In figure 5 , we present the improvement in the octant discovery reach for $0.5^{*} \mathrm{LBNO}$ and LBNE10 with the addition of the projected data from T2K (2.5 years of $\nu$ run and 2.5 years of $\bar{\nu}$ run) and $\mathrm{NO} \nu \mathrm{A}$ (3 years of $\nu$ run and 3 years of $\bar{\nu}$ run). Adding data from current generation experiments helps both $0.5^{*} \mathrm{LBNO}$ and LBNE10 to achieve more than $3 \sigma$ discovery for all true octant-hierarchy- $\delta_{\mathrm{CP}}$ combinations. For $\mathrm{LO}-(\mathrm{NH} / \mathrm{IH})$ true, these setups can provide close to $3.8 \sigma$ discovery for octant irrespective of the choice of true $\delta_{\mathrm{CP}}$.

In the discussion so far, we consider only the best-fit true values of $\sin ^{2} \theta_{23}$ in both the octants. Now, we address the octant resolution capability for values of $\operatorname{true}^{2} \sin ^{2} \theta_{23}$ in the full $3 \sigma$ allowed range of 0.34 to 0.67 . In figure 6 , we plot the $\Delta \chi_{\min }^{2}$ as a function of true $\sin ^{2} \theta_{23}$ for LBNE10 (left panel) and $0.5^{*}$ LBNO (right panel) assuming NH as true hierarchy. Variation of $\delta_{\mathrm{CP}}$ (true) in the range $-180^{\circ}$ to $180^{\circ}$ leads to the band in $\Delta \chi^{2}$ values for a given true $\sin ^{2} \theta_{23}$. The vertical lines point towards the global best-fit values. Here we have added the projected data from $\mathrm{T} 2 \mathrm{~K}$ and $\mathrm{NO} \nu \mathrm{A}$ to produce these results. For LBNE10, a $3 \sigma$ octant resolution is possible for $\sin ^{2} \theta_{23}$ (true) $\leq 0.44$ and for $\sin ^{2} \theta_{23}$ (true) $\geq 0.58$ for all values of $\delta_{\mathrm{CP}}$ (true). For $0.5^{*} \mathrm{LBNO}$, this is possible for $\sin ^{2} \theta_{23}$ (true) $\leq 0.45$ and for $\sin ^{2} \theta_{23}$ (true) $\geq 0.57$. We present the results for IH as true choice in appendix A. 


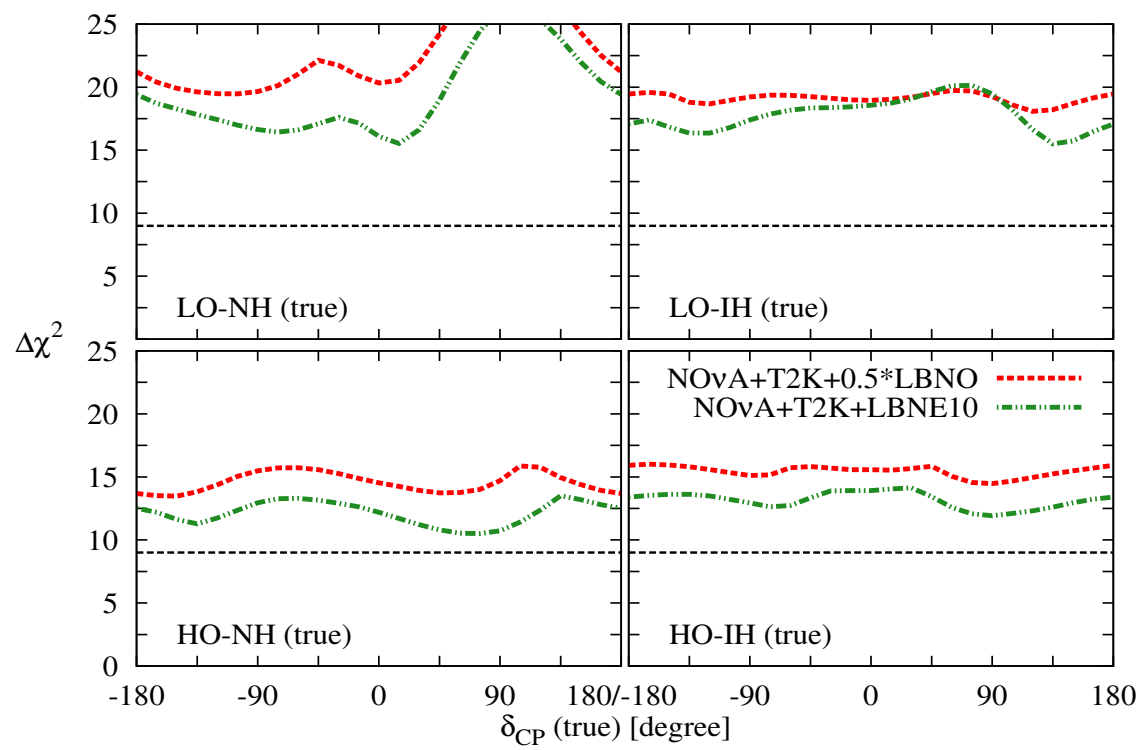

Figure 5. $\Delta \chi_{\min }^{2}$ for octant discovery potential as a function of true $\delta_{\mathrm{CP}}$ for $0.5^{*} \mathrm{LBNO}$ and LBNE10 adding the projected data from $\mathrm{T} 2 \mathrm{~K}$ and $\mathrm{NO} \nu \mathrm{A}$. Results are shown for the four possible true octanthierarchy combinations. For LO $(\mathrm{HO}), \sin ^{2} \theta_{23}($ true $)=0.41(0.59)$. Here $\sin ^{2} 2 \theta_{13}($ true $)=0.089$.
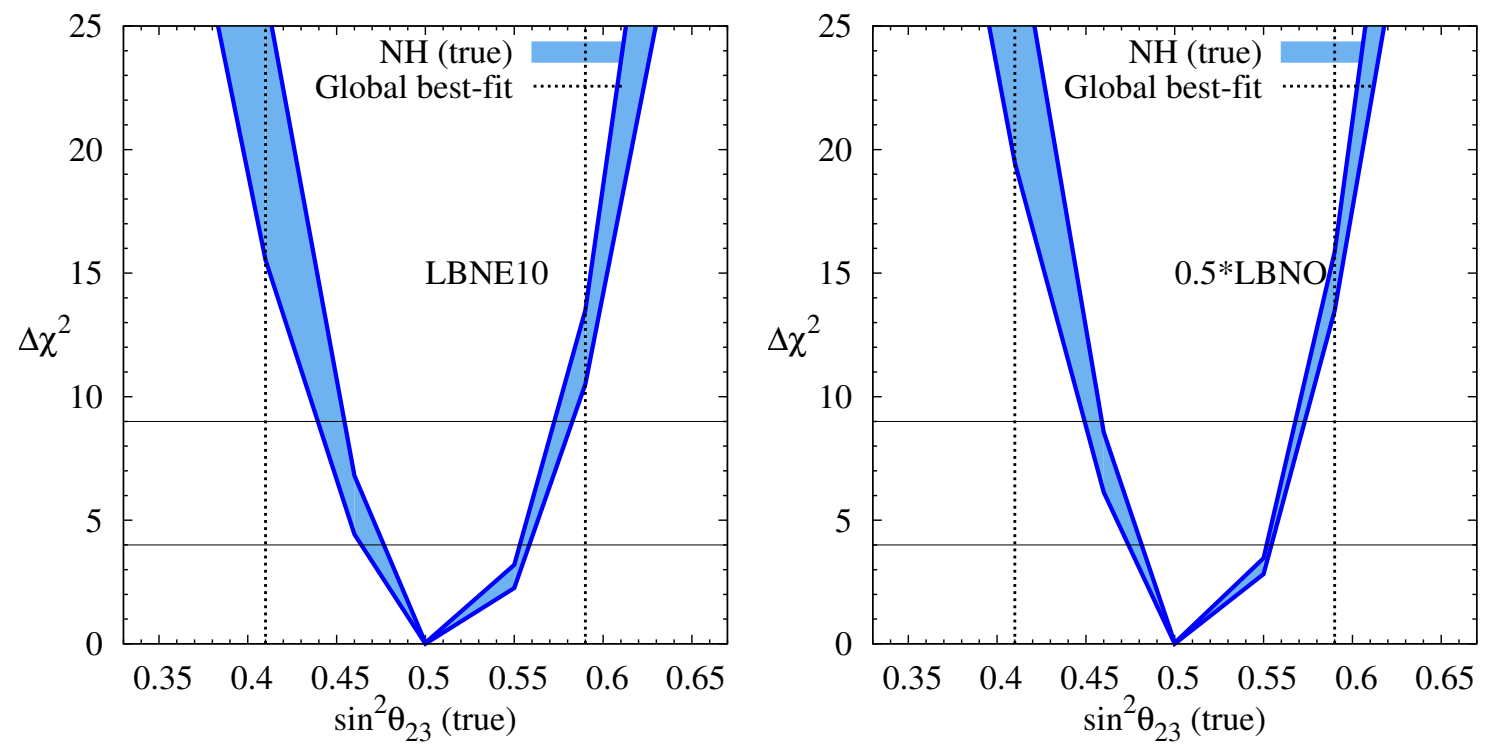

Figure 6. $\Delta \chi_{\min }^{2}$ for octant resolution as a function of true $\sin ^{2} \theta_{23}$. Left panel (right panel) is for LBNE10 $\left(0.5^{*} \mathrm{LBNO}\right)$. The variation due to $\delta_{\mathrm{CP}}$ (true) leads to the band in $\Delta \chi^{2}$ for a given $\sin ^{2} \theta_{23}$ (true). The vertical lines correspond to the global best-fit values. We consider NH as true hierarchy. In producing all these plots, the projected data from $\mathrm{T} 2 \mathrm{~K}$ and $\mathrm{NO} \nu \mathrm{A}$ have been added (see section 3 for details).

Figure 7 depicts the $3 \sigma$ and $5 \sigma$ octant resolution contours in true $\sin ^{2} \theta_{23}-$ true $\delta_{\mathrm{CP}}$ plane assuming $\mathrm{NH}$ as true hierarchy. The left (right) panel is for LBNE10 (0.5*LBNO) 

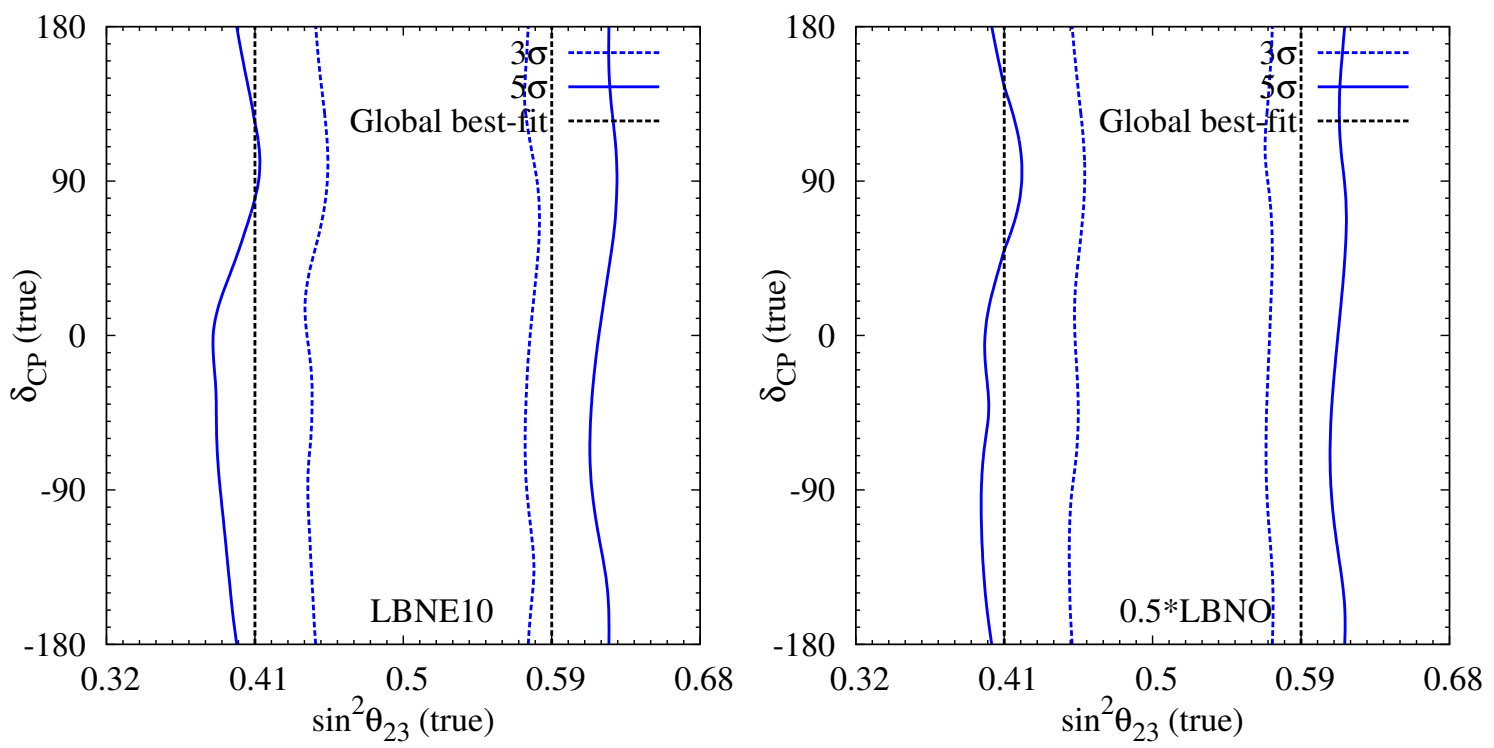

Figure 7. Octant resolving capability at $3 \sigma$ and $5 \sigma$ C.L. in the true $\sin ^{2} \theta_{23}-$ true $\delta_{\text {CP }}$ plane for LBNE10 (left panel) and $0.5^{*}$ LBNO (right panel). The vertical lines point towards the global best-fit values. Here, we assume $\mathrm{NH}$ as true hierarchy. In generating all these plots, the projected data from $\mathrm{T} 2 \mathrm{~K}$ and $\mathrm{NO} \nu \mathrm{A}$ have been added (see section 3 for details).

adding the expected data from $\mathrm{T} 2 \mathrm{~K}$ and $\mathrm{NO} \nu \mathrm{A}$. Octant resolution is only possible for points lying outside the contours. This figure again confirms that both LBNE10 and 0.5*LBNO in combination with $\mathrm{T} 2 \mathrm{~K}$ and $\mathrm{NO} \nu \mathrm{A}$ data can provide octant discovery for global bestfit points at $3 \sigma$ confidence level. We show the similar figure for the true IH choice in appendix A.

Our results agree with figure 4-20 of [36] where results have been shown for a standalone LBNE detector. It can be seen from this figure that a $3 \sigma$ discovery of octant is possible with this set-up if $\theta_{23} \leq 40^{\circ}$ or $\theta_{23} \geq 51^{\circ}$. We achieve somewhat improved sensitivity to octant when the data from $\mathrm{T} 2 \mathrm{~K}$ and $\mathrm{NO} \nu \mathrm{A}$ is combined, as can be seen from figure 6 (left panel) in our work. For LBNO, we present for the first time the results on octant of $\theta_{23}$ and this study has not been performed in the expression of interest for LBNO [41].

\subsection{Discovery reach for leptonic CP violation}

A 'discovery' of leptonic CP violation, if it exists in Nature, means that we can reject both the CP-conserving values of $0^{\circ}, 180^{\circ}$ at a given confidence level. Obviously, this measurement becomes very difficult when $\delta_{\mathrm{CP}}$ approaches to $0^{\circ}, 180^{\circ}$. Therefore, whilst it is possible to discover the mass hierarchy for all possible values of $\delta_{\mathrm{CP}}$, the same is not true in the case of $\mathrm{CP}$ violation study. We have already emphasized that the present uncertainty in the knowledge of $\sin ^{2} \theta_{23}$ has a crucial impact on the discovery reach of mass ordering and octant of $\theta_{23}$ for the experimental setups under consideration. This is also true for the $\mathrm{CP}$ violation discovery reach. We can see from the appearance probability expression in equation $(2.2)$ that both the $\mathrm{CP}$-violating $\left(C_{-}\right)$and $\mathrm{CP}$-conserving $\left(C_{+}\right)$terms depend on $\sin 2 \theta_{23}$, therefore these terms are not sensitive to the octant of $\theta_{23}$ but they depend on 

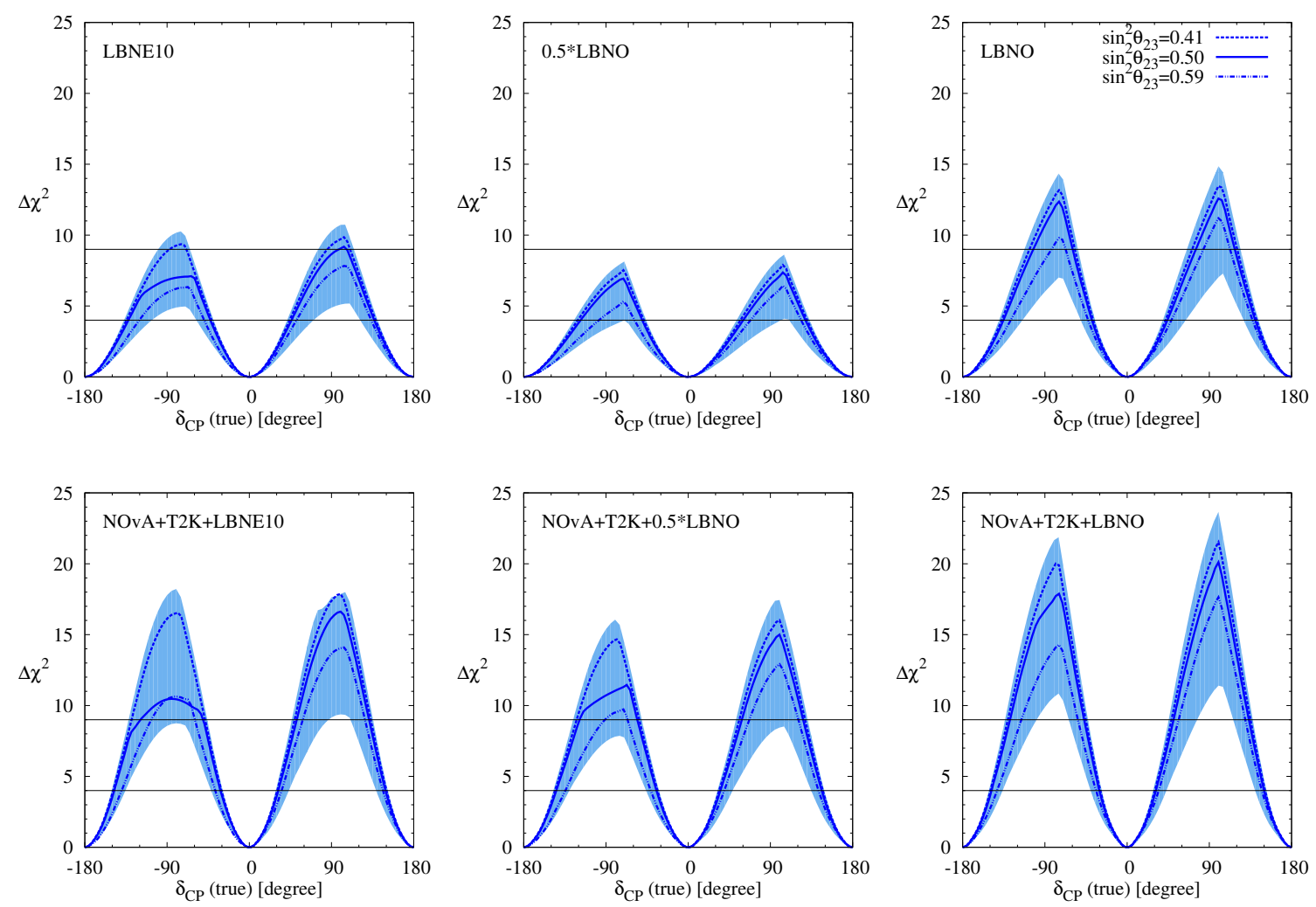

Figure 8. CP Violation discovery reach as a function of true value of $\delta_{\mathrm{CP}}$ assuming $\mathrm{NH}$ as true hierarchy. Results are shown for LBNE10 (10 kt), 0.5*LBNO (10 kt), and LBNO (20 kt) setups in the left, middle, and right upper panels respectively. In lower panels, we show the same including the projected data from $\mathrm{T} 2 \mathrm{~K}$ and $\mathrm{NO} \nu \mathrm{A}$ experiments. The shaded band depicts the variation in $\Delta \chi_{\min }^{2}$ due to different true choices of $\sin ^{2} \theta_{23}$ in its $3 \sigma$ allowed range of 0.34 to 0.67 . Inside the band, we show the results for three different true values of $\sin ^{2} \theta_{23}: 0.41,0.5$, and 0.59 .

the value of $\theta_{23}$. The leading term $\left(C_{0}\right)$ in equation (2.2) is proportional to $\sin ^{2} \theta_{23}$ and therefore it is sensitive to both the octant and magnitude of $\theta_{23}$. In this paper for the first time, we study in detail the $\mathrm{CP}$ violation discovery reach by varying the true value of $\sin ^{2} \theta_{23}$ in its allowed $3 \sigma$ range of 0.34 to 0.67 . We follow the same marginalization scheme in the fit for oscillation parameters and systematic uncertainties as that in the case of mass hierarchy discovery study. For CP violation searches, the final $\Delta \chi^{2}$ is also marginalized over both the choices of hierarchy in the fit to obtain $\Delta \chi_{\min }^{2}$.

In figure 8, we present the $\mathrm{CP}$ violation discovery reach for various experimental setups under consideration as a function of true $\delta_{\mathrm{CP}}$ assuming $\mathrm{NH}$ as true hierarchy. Similar figure for the true $\mathrm{IH}$ choice is given in appendix B. The left, middle, and right upper panels of figure 8 show the results for LBNE10, $0.5^{*} \mathrm{LBNO}$, and LBNO respectively. In lower panels, we depict the same results, combining the projected data from $\mathrm{T} 2 \mathrm{~K}$ and $\mathrm{NO} \nu \mathrm{A}$ experiments. The shaded band in each panel reflects the variation in $\Delta \chi_{\min }^{2}$ due to different true choices of $\sin ^{2} \theta_{23}$ in its $3 \sigma$ allowed range of 0.34 to 0.67 . Inside the band, we give the results for three different true values of $\sin ^{2} \theta_{23}: 0.41,0.5$, and 0.59 . We summarize the 
main features of figure 8 in table 1 . In their first phases, both LBNE10 and LBNO will have CP violation reach for around $50 \%$ values of true $\delta_{\mathrm{CP}}$ at $2 \sigma$ confidence level (see table 1 ). At $3 \sigma$, their CP violation reach is quite minimal: only $3 \%$ for LBNE10 and $23 \%$ for LBNO. It is quite important to note that the addition of the projected $\mathrm{T} 2 \mathrm{~K}$ and $\mathrm{NO} \nu \mathrm{A}$ data helps a lot to improve the $\mathrm{CP}$ coverage for these setups at $3 \sigma$ confidence level for all possible true values of $\sin ^{2} \theta_{23}$ (see figure 8). For an example, LBNE10 (LBNO) can achieve CP violation discovery for $43 \%(46 \%)$ values of true $\delta_{\mathrm{CP}}$ at $3 \sigma$ combining the expected data from the current generation experiments $\mathrm{T} 2 \mathrm{~K}$ and $\mathrm{NO} \nu \mathrm{A}$ assuming $\sin ^{2} \theta_{23}$ (true) $=0.5$. For $0.5^{*} \mathrm{LBNO}$, we do not have any sensitivity at $3 \sigma$ C.L. but, adding the $\mathrm{T} 2 \mathrm{~K}$ and $\mathrm{NO} \nu \mathrm{A}$ data, $37 \% \mathrm{CP}$ coverage can be obtained. We see that the $\mathrm{CP}$ violation discovery reach of LBNE10 is better than that of $0.5^{*} \mathrm{LBNO}$ and is comparable to that of LBNO (whose detector mass is $20 \mathrm{kt}$ and is two times larger compared to LBNE10). The reason for this is three fold: (a) the shorter baseline leads to more flux at the detector, (b) the percentage change in $\nu_{\mu} \rightarrow \nu_{e}$ oscillation channel with respect to $\delta_{\mathrm{CP}}$ is more at lower energies, and (c) the $\mathrm{CP}$ asymmetry due to $\delta_{\mathrm{CP}}$ is less swamped by the fake $\mathrm{CP}$ asymmetry due to the matter potential. It was shown in figure 17 of [70] that a baseline close to $1300 \mathrm{~km}$ is optimal for the discovery of $\mathrm{CP}$ violation for large $\theta_{13}$.

All these results again clearly demonstrate that the projected data from the current generation off-axis superbeam experiments will be quite useful for future generation on-axis wide band superbeam setups to enhance their discovery reach at higher confidence level. Another important feature that emerges from figure 8 is that the $\mathrm{CP}$ violation discovery reach is quite sensitive to the true value of $\sin ^{2} \theta_{23}$. The results are better if $\sin ^{2} \theta_{23}$ (true) belongs to LO compared to HO. The main reason behind this is that like in the case of $\theta_{13}[72,73]$, the CP-asymmetry increases if we lower the value of $\theta_{23}$, reducing the strength of the leading term $\left(C_{0}\right)$ in equation (2.2).

Our results for $\mathrm{CP}$ violation discovery reach in the case of LBNE10 are quite similar to those presented in the lower panels of figure $4-13$ in [36]. Here, one can see a $58 \%$ coverage in true $\delta_{\mathrm{CP}}$ at $2 \sigma$ confidence level. For $0.5^{*} \mathrm{LBNO}$, the results are a little worse compared to LBNE10. Figure 75 in reference [41] shows that LBNO with a $20 \mathrm{kt}$ detector has similar sensitivity as that of LBNE10. Here also, a $58 \%$ coverage in true $\delta_{\mathrm{CP}}$ is achieved at $2 \sigma$ confidence level.

\section{Concluding remarks}

With the recent measurement of $\theta_{13}$ by reactor experiments, a clear and comprehensive picture of the three flavor leptonic mixing matrix has been established. This impressive result has crucial consequences for future theoretical and experimental efforts. It has opened up the possibility to probe the sub-dominant three flavor effects in both current and future long-baseline oscillation facilities. Another interesting piece of information on $\theta_{23}$ has been provided by recently completed MINOS accelerator experiment. $\nu_{\mu} \rightarrow \nu_{\mu}$ disappearance data of MINOS points towards the deviation from maximal 2-3 mixing, causing the octant ambiguity of $\theta_{23}$. In this paper, we present a comparative study of the physics reach of two future superbeam facilities, LBNE and LBNO in their first phases of run, in address- 


\begin{tabular}{|c|c|c|}
\hline \multirow{2}{*}{ Setups } & \multicolumn{2}{|c|}{ Fraction of $\delta_{\mathrm{CP}}($ true $)$} \\
\cline { 2 - 3 } & $2 \sigma$ confidence level & $3 \sigma$ confidence level \\
\hline LBNE10 $(10 \mathrm{kt})$ & 0.51 & 0.03 \\
\hline LBNE10 $+\mathrm{T} 2 \mathrm{~K}+\mathrm{NO} \nu \mathrm{A}$ & 0.63 & 0.43 \\
\hline $0.5^{*} \mathrm{LBNO}(10 \mathrm{kt})$ & 0.40 & 0.0 \\
\hline $0.5 * \mathrm{LBNO}+\mathrm{T} 2 \mathrm{~K}+\mathrm{NO} \nu \mathrm{A}$ & 0.63 & 0.37 \\
\hline $\mathrm{LBNO}(20 \mathrm{kt})$ & 0.51 & 0.23 \\
\hline $\mathrm{LBNO}+\mathrm{T} 2 \mathrm{~K}+\mathrm{NO} \nu \mathrm{A}$ & 0.69 & 0.46 \\
\hline
\end{tabular}

Table 1. Fraction of $\delta_{\mathrm{CP}}$ (true) for which a discovery is possible for $\mathrm{CP}$ violation considering $\mathrm{NH}$ as true hierarchy. Here, we assume maximal mixing for the true choice of $\theta_{23}$. The results are presented at $2 \sigma$ and $3 \sigma$ confidence level.

ing the issues of neutrino mass hierarchy, octant of $\theta_{23}$, and leptonic $\mathrm{CP}$ violation. We also demonstrate that the projected data from current generation experiments, $\mathrm{T} 2 \mathrm{~K}$ and $\mathrm{NO} \nu \mathrm{A}$ will play a crucial role for these future facilities to achieve their milestones with higher confidence level. Also for the first time, we study in detail the impact of the present uncertainty in 2-3 mixing angle in resolving these fundamental issues.

We find that in its first phase, even a 50\% scaled down version of LBNO with $10 \mathrm{kt}$ detector mass has more than $7 \sigma$ mass hierarchy discovery reach for the lowest possible value of $\sin ^{2} \theta_{23}$ (true) $=0.34$ in its presently allowed $3 \sigma$ range. This result is valid for any choices of true $\delta_{\mathrm{CP}}$ and hierarchy. However, LBNE10 suffers in this regard and will not be able to provide a $5 \sigma$ result for about $50 \%$ of the true $\delta_{\mathrm{CP}}$ range even for maximal mixing choice for $\sin ^{2} \theta_{23}$ (true). Moreover, it fails to achieve even a $3 \sigma$ hierarchy discovery for the best-fit value in $\mathrm{LO}, \sin ^{2} \theta_{23}$ (true) $=0.41$ and the worst case combinations of the true parameters $\left(\mathrm{NH}, 90^{\circ}\right)$ and $\left(\mathrm{IH},-90^{\circ}\right)$. In such a scenario, the projected data from $\mathrm{T} 2 \mathrm{~K}$ and $\mathrm{NO} \nu \mathrm{A}$ can be extremely useful for LBNE10. Adding the expected informations from T2K and $\mathrm{NO} \nu \mathrm{A}, \mathrm{LBNE} 10$ can discover mass hierarchy at $3 \sigma$ confidence level for any combinations of true hierarchy- $\delta_{\mathrm{CP}}$ and even for the most conservative choice of $\sin ^{2} \theta_{23}$ (true) $=0.34$ in its present $3 \sigma$ range. It clearly corroborates the synergy between the current (off-axis) and future (on-axis) superbeam experiments.

As far as the octant discovery is concerned, adding the projected data from equal neutrino and anti-neutrino runs of $\mathrm{T} 2 \mathrm{~K}$ (2.5 years each) and $\mathrm{NO} \nu \mathrm{A}$ ( 3 years each), LBNE10 can provide a $3 \sigma$ octant resolution for $\sin ^{2} \theta_{23}$ (true) $\leq 0.44$ and for $\sin ^{2} \theta_{23}$ (true) $\geq 0.58$ for all values of $\delta_{\mathrm{CP}}$ (true). For $0.5^{*} \mathrm{LBNO}$, this is possible for $\sin ^{2} \theta_{23}$ (true) $\leq 0.45$ and for $\sin ^{2} \theta_{23}$ (true) $\geq 0.57$.

In their first phases, both LBNE10 and LBNO can establish leptonic CP violation for around $50 \%$ values of true $\delta_{\mathrm{CP}}$ at $2 \sigma$ confidence level. At $3 \sigma$, their $\mathrm{CP}$ violation reach is quite minimal: only $3 \%$ for LBNE10 and $23 \%$ for LBNO. The expected measurements from present generation experiments $\mathrm{T} 2 \mathrm{~K}$ and $\mathrm{NO} \nu \mathrm{A}$ can have dramatic impact on the $\mathrm{CP}$ violation discovery reach of the future facilities in their first phases of run. In case of 

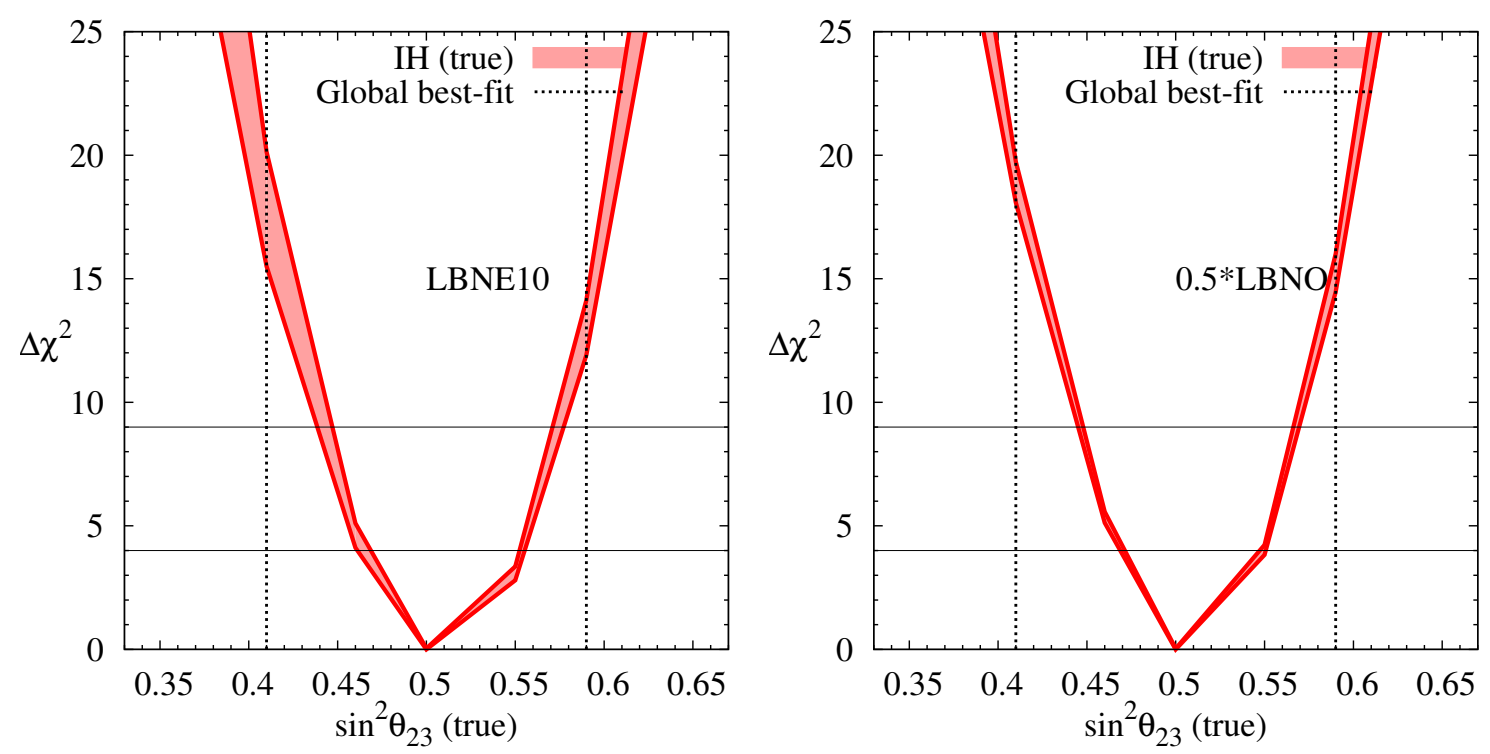

Figure 9. $\Delta \chi_{\min }^{2}$ for octant resolution as a function of true $\sin ^{2} \theta_{23}$. Left panel (right panel) is for LBNE10 $(0.5 * \mathrm{LBNO})$. The variation due to $\delta_{\mathrm{CP}}$ (true) leads to the band in $\Delta \chi^{2}$ for a given $\sin ^{2} \theta_{23}$ (true). The vertical lines correspond to the global best-fit values. We consider IH as true hierarchy. In producing all these plots, the projected data from $\mathrm{T} 2 \mathrm{~K}$ and $\mathrm{NO} \nu \mathrm{A}$ have been added (see section 3 for details).

LBNE10, CP coverage can be enhanced from $3 \%$ to $43 \%$ at $3 \sigma$ combining $\mathrm{T} 2 \mathrm{~K}$ and $\mathrm{NO} \nu \mathrm{A}$ data assuming $\sin ^{2} \theta_{23}$ (true) $=0.5$. For LBNO setup, CP violation discovery is possible for $46 \%$ values of true $\delta_{\mathrm{CP}}$ at $3 \sigma$ if we add the data from $\mathrm{T} 2 \mathrm{~K}$ and $\mathrm{NO} \nu \mathrm{A}$.

\section{Acknowledgments}

We would like to thank M. Bishai, G. Zeller, A. Rubbia and M. Goodman for helpful communications. SKA was supported by the DST/INSPIRE Research Grant [IFA-PH-12], Department of Science and Technology, India.

\section{A Resolution of octant as a function of true $\theta_{23}$ for IH(true)}

In this appendix, we present the results for octant discovery generating the data with IH. In figure 9 , we show the $\Delta \chi_{\min }^{2}$ as a function of true $\sin ^{2} \theta_{23}$ for LBNE10 (left panel) and $0.5^{*} \mathrm{LBNO}$ (right panel) assuming IH as true hierarchy. Variation of $\delta_{\mathrm{CP}}$ (true) in the range $-180^{\circ}$ to $180^{\circ}$ leads to the band in $\Delta \chi^{2}$ values for a given true $\sin ^{2} \theta_{23}$. The vertical lines indicate towards the global best-fit values. Here we add the projected data from T2K and $\mathrm{NO} \nu \mathrm{A}$ to produce these results. For LBNE10, a $3 \sigma$ octant resolution is possible for $\sin ^{2} \theta_{23}$ (true) $\leq 0.44$ and for $\sin ^{2} \theta_{23}$ (true) $\geq 0.58$ irrespective of the values of $\delta_{\mathrm{CP}}$ (true). For $0.5 *$ LBNO, this is possible for $\sin ^{2} \theta_{23}$ (true) $\leq 0.44$ and for $\sin ^{2} \theta_{23}$ (true) $\geq 0.57$. We see that the results with $\mathrm{IH}$ (true) choice are quite similar to that of $\mathrm{NH}$ (true) (see figure 6).

Figure 10 shows the $3 \sigma$ and $5 \sigma$ octant resolution contours in true $\sin ^{2} \theta_{23}-$ true $\delta_{\mathrm{CP}}$ plane considering IH as true hierarchy. The left (right) panel is for LBNE10 (0.5*LBNO) 

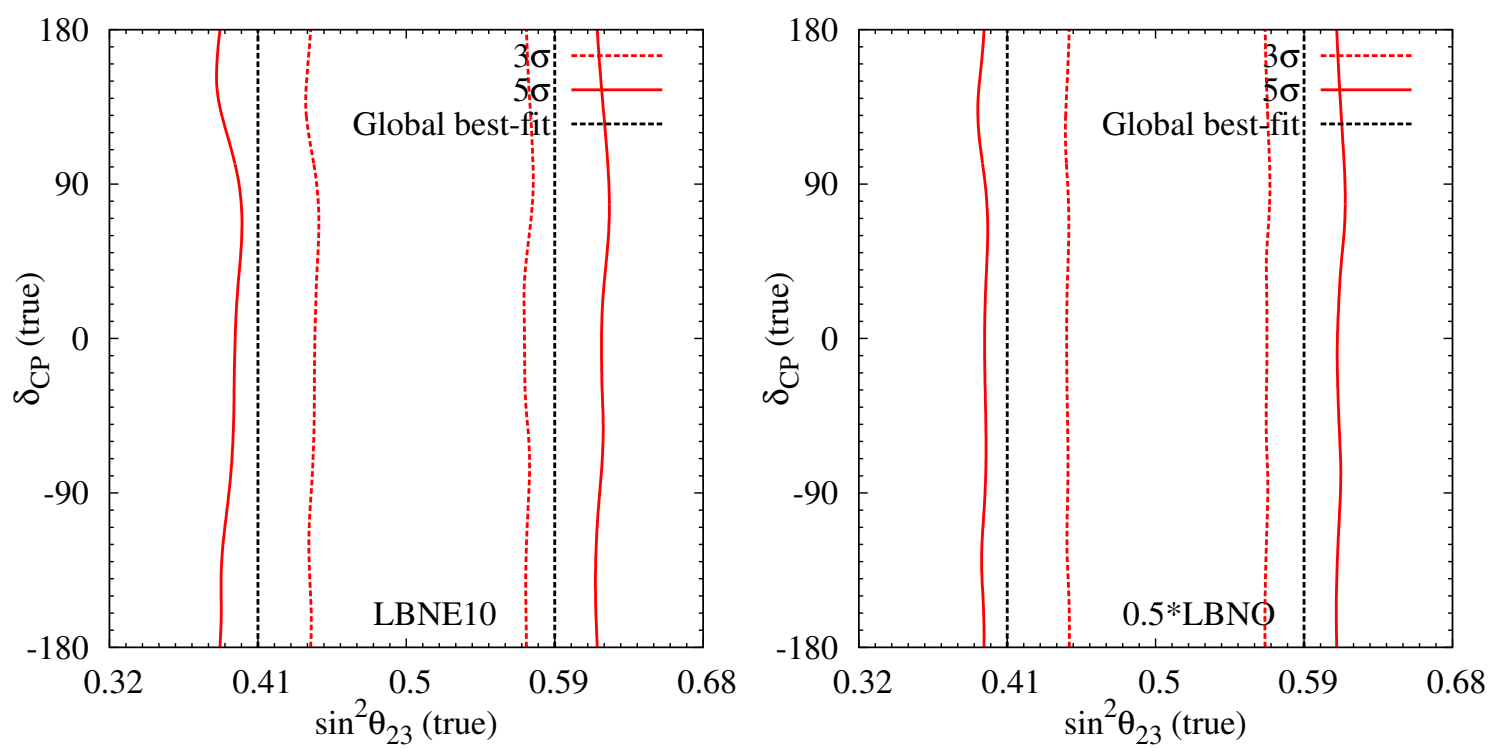

Figure 10. Octant resolving capability at $3 \sigma$ and $5 \sigma$ C.L. in the true $\sin ^{2} \theta_{23}-$ true $\delta_{\text {CP }}$ plane for LBNE10 (left panel) and $0.5^{*}$ LBNO (right panel). The vertical lines point towards the global best-fit values. Here, we assume IH as true hierarchy. In generating all these plots, the projected data from $\mathrm{T} 2 \mathrm{~K}$ and $\mathrm{NO} \nu \mathrm{A}$ have been added (see section 3 for details).

adding the expected data from $\mathrm{T} 2 \mathrm{~K}$ and $\mathrm{NO} \nu \mathrm{A}$. Octant resolution is only possible for points lying outside the contours. This figure again suggests that for $\mathrm{IH}$ (true) case, both LBNE10 and $0.5^{*} \mathrm{LBNO}$ in combination with $\mathrm{T} 2 \mathrm{~K}$ and $\mathrm{NO} \nu \mathrm{A}$ data can provide octant discovery for global best-fit points at $3 \sigma$ confidence level.

\section{B CP violation discovery as a function of true $\delta_{\mathrm{CP}}$ for $\mathrm{IH}($ true)}

In figure 11, we give the $\mathrm{CP}$ violation discovery reach for various experimental setups under study as a function of true $\delta_{\mathrm{CP}}$ considering IH as true hierarchy. Like in figure 8 , the left, middle, and right upper panels of figure 11 present the results for LBNE10, 0.5*LBNO, and LBNO respectively. In lower panels, we depict the same combining the projected data from $\mathrm{T} 2 \mathrm{~K}$ and $\mathrm{NO} \nu \mathrm{A}$ experiments. The shaded band in each panel reflects the variation in $\Delta \chi_{\min }^{2}$ due to different true choices of $\sin ^{2} \theta_{23}$ in its $3 \sigma$ allowed range of 0.34 to 0.67 . Inside the band, we give the results for three different true values of $\sin ^{2} \theta_{23}: 0.41,0.5$, and 0.59 . We do not see any qualitative differences in the $\mathrm{CP}$ violation discovery reach for these setups when we generate the data assuming IH instead of NH. Around $\delta_{\mathrm{CP}}($ true $)= \pm 90^{\circ}$ (where $\mathrm{CP}$ violation is maximum), the results are slightly better for $\mathrm{NH}$ (true) compared to $\mathrm{IH}$ (true).

Open Access. This article is distributed under the terms of the Creative Commons Attribution License (CC-BY 4.0), which permits any use, distribution and reproduction in any medium, provided the original author(s) and source are credited. 

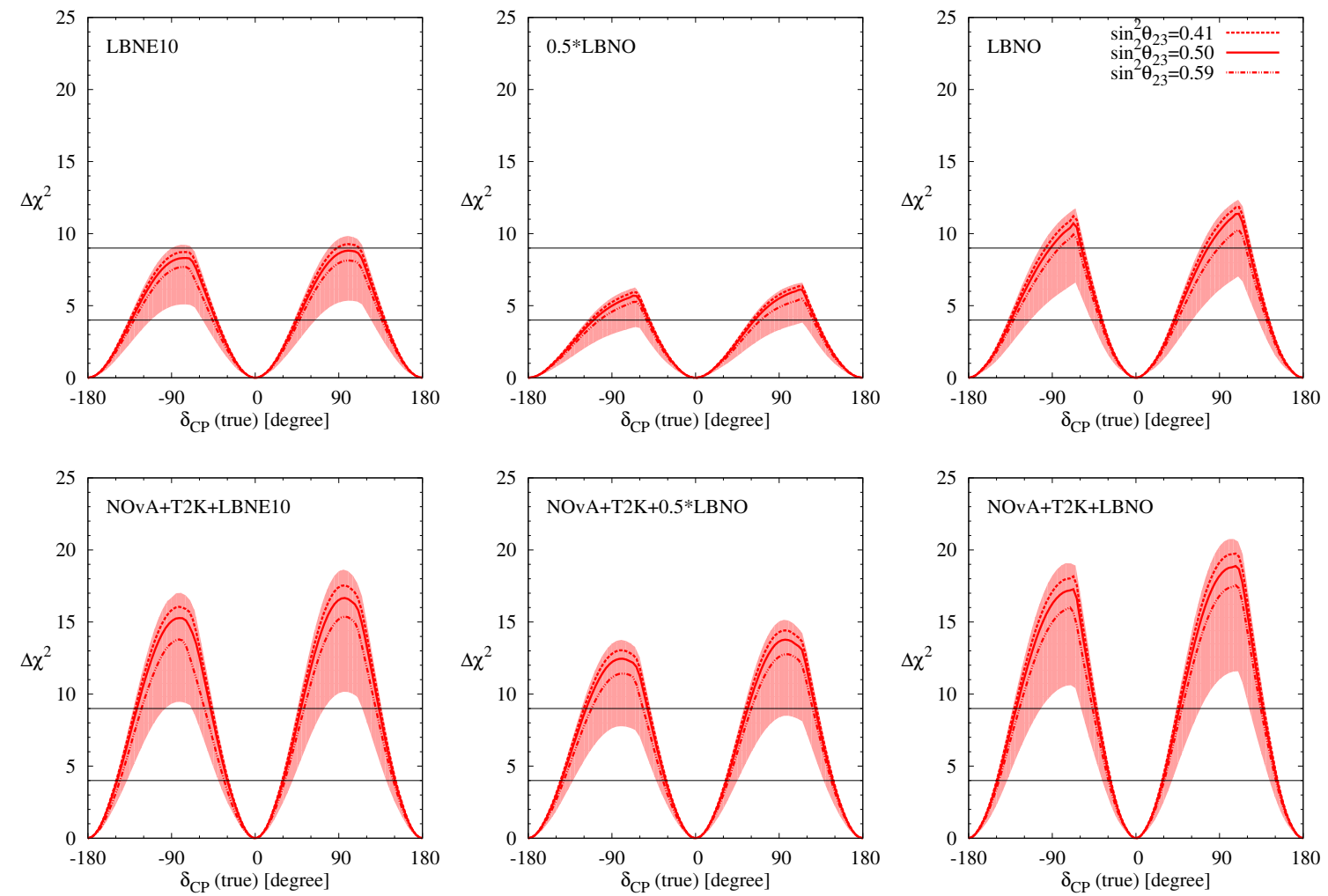

Figure 11. CP Violation discovery reach as a function of true value of $\delta_{\mathrm{CP}}$ assuming IH as true hierarchy. Results are shown for LBNE10 (10 kt), 0.5*LBNO (10 kt), and LBNO (20 kt) in the left, middle, and right upper panels respectively. In lower panels, we depict the same adding the information from $\mathrm{T} 2 \mathrm{~K}$ and $\mathrm{NO} \nu \mathrm{A}$ experiments. The shaded band depicts the variation in $\Delta \chi_{\min }^{2}$ due to different true choices of $\sin ^{2} \theta_{23}$ in its $3 \sigma$ allowed range of 0.34 to 0.67 . Inside the band, we show the results for three different true values of $\sin ^{2} \theta_{23}: 0.41,0.5$, and 0.59 .

\section{References}

[1] Daya BAY collaboration, F.P. An et al., Spectral measurement of electron antineutrino oscillation amplitude and frequency at Daya Bay, Phys. Rev. Lett. 112 (2014) 061801 [arXiv: 1310.6732] [INSPIRE].

[2] DAYA-BAY collaboration, F.P. An et al., Observation of electron-antineutrino disappearance at Daya Bay, Phys. Rev. Lett. 108 (2012) 171803 [arXiv:1203.1669] [INSPIRE].

[3] RENO collaboration, J.K. Ahn et al., Observation of Reactor Electron Antineutrino Disappearance in the RENO Experiment, Phys. Rev. Lett. 108 (2012) 191802 [arXiv: 1204.0626] [INSPIRE].

[4] Double CHOOZ collaboration, Y. Abe et al., Reactor electron antineutrino disappearance in the Double CHOOZ experiment, Phys. Rev. D 86 (2012) 052008 [arXiv:1207.6632] [INSPIRE].

[5] R. Nichol, New Results from MINOS, Nucl. Phys. B Proc. Suppl. 235 (2013) 105. 
[6] MINOS collaboration, P. Adamson et al., Measurement of Neutrino and Antineutrino Oscillations Using Beam and Atmospheric Data in MINOS, Phys. Rev. Lett. 110 (2013) 251801 [arXiv: 1304.6335] [INSPIRE].

[7] KATRIN collaboration, A. Osipowicz et al., KATRIN: a Next generation tritium beta decay experiment with sub-eV sensitivity for the electron neutrino mass. Letter of intent, hep-ex/0109033 [INSPIRE].

[8] I. Avignone, Frank T., S.R. Elliott and J. Engel, Double Beta Decay, Majorana Neutrinos and Neutrino Mass, Rev. Mod. Phys. 80 (2008) 481 [arXiv:0708.1033] [INSPIRE].

[9] J. Lesgourgues and S. Pastor, Neutrino mass from Cosmology, Adv. High Energy Phys. 2012 (2012) 608515 [arXiv:1212.6154] [INSPIRE].

[10] Planck collaboration, P.A.R. Ade et al., Planck 2013 results. XVI. Cosmological parameters, arXiv: 1303.5076 [INSPIRE].

[11] NuFIT webpage, http://www.nu-fit.org/.

[12] M.C. Gonzalez-Garcia, M. Maltoni, J. Salvado and T. Schwetz, Global fit to three neutrino mixing: critical look at present precision, JHEP 12 (2012) 123 [arXiv:1209.3023] [INSPIRE].

[13] F. Capozzi, G.L. Fogli, E. Lisi, A. Marrone, D. Montanino et al., Status of three-neutrino oscillation parameters, circa 2013, arXiv:1312.2878 [INSPIRE].

[14] G.L. Fogli and E. Lisi, Tests of three flavor mixing in long baseline neutrino oscillation experiments, Phys. Rev. D 54 (1996) 3667 [hep-ph/9604415] [INSPIRE].

[15] C.H. Albright and M.-C. Chen, Model Predictions for Neutrino Oscillation Parameters, Phys. Rev. D 74 (2006) 113006 [hep-ph/0608137] [INSPIRE].

[16] S. Pascoli, S.T. Petcov and T. Schwetz, The Absolute neutrino mass scale, neutrino mass spectrum, Majorana CP-violation and neutrinoless double-beta decay, Nucl. Phys. B 734 (2006) 24 [hep-ph/0505226] [INSPIRE].

[17] M. Fukugita and T. Yanagida, Baryogenesis Without Grand Unification, Phys. Lett. B 174 (1986) 45 [INSPIRE].

[18] R.N. Mohapatra and S. Nussinov, Bimaximal neutrino mixing and neutrino mass matrix, Phys. Rev. D 60 (1999) 013002 [hep-ph/9809415] [InSPIRE].

[19] K.S. Babu, E. Ma and J.W.F. Valle, Underlying $A_{4}$ symmetry for the neutrino mass matrix and the quark mixing matrix, Phys. Lett. B 552 (2003) 207 [hep-ph/0206292] [INSPIRE].

[20] H. Minakata and A.Y. Smirnov, Neutrino mixing and quark-lepton complementarity, Phys. Rev. D 70 (2004) 073009 [hep-ph/0405088] [INSPIRE].

[21] L.J. Hall, H. Murayama and N. Weiner, Neutrino mass anarchy, Phys. Rev. Lett. 84 (2000) 2572 [hep-ph/9911341] [INSPIRE].

[22] A. de Gouvêa and H. Murayama, Neutrino Mixing Anarchy: alive and Kicking, arXiv: 1204.1249 [INSPIRE].

[23] T2K collaboration, Y. Itow et al., The JHF-Kamioka neutrino project, hep-ex/0106019 [INSPIRE].

[24] T2K collaboration, K. Abe et al., The T2K Experiment, Nucl. Instrum. Meth. A 659 (2011) 106 [arXiv: 1106.1238] [INSPIRE].

[25] D. Ayres, G. Drake, M. Goodman, V. Guarino, T. Joffe-Minor et al., Letter of Intent to build an Off-axis Detector to study numu to nue oscillations with the NuMI Neutrino Beam, hep-ex/0210005 [INSPIRE]. 
[26] NOvA collaboration, D.S. Ayres et al., NOvA: proposal to build a 30 kiloton off-axis detector to study $\nu_{\mu} \rightarrow \nu_{e}$ oscillations in the NuMI beamline, hep-ex/0503053 [INSPIRE].

[27] NOvA collaboration, D. Ayres et al., The NOvA Technical Design Report, FERMILAB-DESIGN-2007-01.

[28] P. Huber, M. Lindner, T. Schwetz and W. Winter, First hint for CP-violation in neutrino oscillations from upcoming superbeam and reactor experiments, JHEP 11 (2009) 044 [arXiv:0907.1896] [INSPIRE].

[29] S.K. Agarwalla, S. Prakash, S.K. Raut and S.U. Sankar, Potential of optimized NOvA for large $\theta_{13} \&$ combined performance with a LArTPC \& T2K, JHEP 12 (2012) 075 [arXiv: 1208.3644] [INSPIRE].

[30] S.K. Agarwalla, S. Prakash and S.U. Sankar, Resolving the octant of $\theta_{23}$ with T2K and NOvA, JHEP 07 (2013) 131 [arXiv:1301.2574] [INSPIRE].

[31] A. Chatterjee, P. Ghoshal, S. Goswami and S.K. Raut, Octant sensitivity for large $\theta_{13}$ in atmospheric and long baseline neutrino experiments, JHEP 06 (2013) 010 [arXiv: 1302.1370] [INSPIRE].

[32] M.V. Diwan, D. Beavis, M.-C. Chen, J. Gallardo, S. Kahn et al., Very long baseline neutrino oscillation experiments for precise measurements of mixing parameters and CP-violating effects, Phys. Rev. D 68 (2003) 012002 [hep-ph/0303081] [InSPIRE].

[33] V. Barger, M. Bishai, D. Bogert, C. Bromberg, A. Curioni et al., Report of the US long baseline neutrino experiment study, arXiv:0705.4396 [INSPIRE].

[34] P. Huber and J. Kopp, Two experiments for the price of one? - The role of the second oscillation maximum in long baseline neutrino experiments, JHEP 03 (2011) 013 [Erratum ibid. 1105 (2011) 024] [arXiv:1010.3706] [INSPIRE].

[35] LBNE collaboration, T. Akiri et al., The 2010 Interim Report of the Long-Baseline Neutrino Experiment Collaboration Physics Working Groups, arXiv:1110.6249 [INSPIRE].

[36] LBNE collaboration, C. Adams et al., Scientific Opportunities with the Long-Baseline Neutrino Experiment, arXiv:1307.7335 [INSPIRE].

[37] D. Autiero, J. Aysto, A. Badertscher, L.B. Bezrukov, J. Bouchez et al., Large underground, liquid based detectors for astro-particle physics in Europe: scientific case and prospects, JCAP 11 (2007) 011 [arXiv:0705.0116] [INSPIRE].

[38] A. Rubbia, A CERN-Based High-Intensity High-Energy Proton Source for Long baseline Neutrino Oscillation Experiments with Next-Generation Large Underground detectors for Proton Decay Searches and Neutrino Physics and Astrophysics, arXiv:1003.1921 [INSPIRE].

[39] LAGUNA collaboration, D. Angus et al., The LAGUNA design study-towards giant liquid based underground detectors for neutrino physics and astrophysics and proton decay searches,

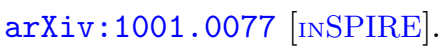

[40] LAGUNA collaboration, A. Rubbia, The LAGUNA design study: towards giant liquid based underground detectors for neutrino physics and astrophysics and proton decay searches, Acta Phys. Polon. B 41 (2010) 1727 [InSPIRE].

[41] A. Stahl, C. Wiebusch, A. Guler, M. Kamiscioglu, R. Sever et al., Expression of Interest for a very long baseline neutrino oscillation experiment (LBNO), CERN-SPSC-2012-021.

[42] L. Wolfenstein, Neutrino Oscillations in Matter, Phys. Rev. D 17 (1978) 2369 [InSPIRE]. 
[43] S.P. Mikheev and A.Y. Smirnov, Resonance Amplification of Oscillations in Matter and Spectroscopy of Solar Neutrinos, Sov. J. Nucl. Phys. 42 (1985) 913 [Yad. Fiz. 42 (1985) 1441] [INSPIRE].

[44] V.D. Barger, K. Whisnant, S. Pakvasa and R.J.N. Phillips, Matter Effects on Three-Neutrino Oscillations, Phys. Rev. D 22 (1980) 2718 [InSPIRE].

[45] A. Cervera, A. Donini, M.B. Gavela, J.J. Gomez Cadenas, P. Hernández et al., Golden measurements at a neutrino factory, Nucl. Phys. B 579 (2000) 17 [Erratum ibid. B 593 (2001) 731] [hep-ph/0002108] [INSPIRE].

[46] M. Freund, P. Huber and M. Lindner, Systematic exploration of the neutrino factory parameter space including errors and correlations, Nucl. Phys. B 615 (2001) 331 [hep-ph/0105071] [INSPIRE].

[47] E.K. Akhmedov, R. Johansson, M. Lindner, T. Ohlsson and T. Schwetz, Series expansions for three flavor neutrino oscillation probabilities in matter, JHEP 04 (2004) 078 [hep-ph/0402175] [INSPIRE].

[48] H. Minakata and H. Nunokawa, Exploring neutrino mixing with low-energy superbeams, JHEP 10 (2001) 001 [hep-ph/0108085] [INSPIRE].

[49] V. Barger, D. Marfatia and K. Whisnant, Breaking eight fold degeneracies in neutrino CP-violation, mixing and mass hierarchy, Phys. Rev. D 65 (2002) 073023 [hep-ph/0112119] [INSPIRE].

[50] A. Para and M. Szleper, Neutrino oscillations experiments using off-axis NuMI beam, hep-ex/0110032 [INSPIRE].

[51] T2K collaboration, K. McFarland et al., Oscillation Results from T2K, talk given at Fermilab Joint Experimental-Theoretical Semiar, August 23, 2013, Fermilab, U.S.A., http://theory.fnal.gov/jetp/talks/T2K-McFarland-Fermilab-23August2013.pdf.

[52] M. Fechner, Détermination des performances attendues sur la recherche de l'oscillation $\nu_{\mu} \rightarrow \nu_{e}$ dans l'expérience T2K depuis l'étude des données recueillies dans l'expérience K2K, DAPNIA-2006-01-T.

[53] NOvA collaboration, R.B. Patterson, The NOvA Experiment: status and Outlook, Nucl. Phys. Proc. Suppl. 235 (2013) 151 [arXiv:1209.0716] [InSPIRE].

[54] Mary Bishai, private communication, 2012.

[55] S.K. Agarwalla, T. Li and A. Rubbia, An Incremental approach to unravel the neutrino mass hierarchy and CP-violation with a long-baseline Superbeam for large $\theta_{13}, J H E P 05$ (2012) 154 [arXiv: 1109.6526] [INSPIRE].

[56] Geralyn Zeller, private communication, 2012.

[57] R. Petti and G. Zeller, Nuclear Effects in Water vs. Argon, Tech. Rep. LBNE docdb, no. 740.

[58] Silvestro di Luise, Optimization of Neutrino Fluxes for Future Long Baseline Neutrino Experiment, poster presented at the ICHEP2012 Conference, July 4-11 2012, Melbourne, Australia, http://www.ichep2012.com.au/.

[59] P. Huber, M. Lindner and W. Winter, Simulation of long-baseline neutrino oscillation experiments with GLoBES (General Long Baseline Experiment Simulator), Comput. Phys. Commun. 167 (2005) 195 [hep-ph/0407333] [INSPIRE].

[60] P. Huber, J. Kopp, M. Lindner, M. Rolinec and W. Winter, New features in the simulation of neutrino oscillation experiments with GLoBES 3.0: general Long Baseline Experiment Simulator, Comput. Phys. Commun. 177 (2007) 432 [hep-ph/0701187] [INSPIRE]. 
[61] H. Nunokawa, S.J. Parke and R. Zukanovich Funchal, Another possible way to determine the neutrino mass hierarchy, Phys. Rev. D 72 (2005) 013009 [hep-ph/0503283] [INSPIRE].

[62] A. de Gouvêa, J. Jenkins and B. Kayser, Neutrino mass hierarchy, vacuum oscillations and vanishing $|\mathrm{U}(\mathrm{e} 3)|$, Phys. Rev. D 71 (2005) 113009 [hep-ph/0503079] [INSPIRE].

[63] M. Blennow, P. Coloma, A. Donini and E. Fernandez-Martinez, Gain fractions of future neutrino oscillation facilities over T2K and NOvA, JHEP 07 (2013) 159 [arXiv:1303.0003] [INSPIRE].

[64] DAYA BAY collaboration, X. Qian et al., Improved Measurement of Electron-antineutrino Disappearance at Daya Bay, talk given at the NuFact 2012 Conference, July 23-28 2012, Williamsburg, U.S.A., http://www.jlab.org/conferences/nufact12/.

[65] MINERvA collaboration, L. Aliaga et al., Design, Calibration and Performance of the MINERvA Detector, Nucl. Instrum. Meth. A 743 (2014) 130 [arXiv:1305.5199] [INSPIRE].

[66] P. Coloma, P. Huber, J. Kopp and W. Winter, Systematic uncertainties in long-baseline neutrino oscillations for large $\theta_{13}$, Phys. Rev. D 87 (2013) 033004 [arXiv:1209.5973] [INSPIRE].

[67] M. Blennow, P. Coloma, P. Huber and T. Schwetz, Quantifying the sensitivity of oscillation experiments to the neutrino mass ordering, arXiv:1311.1822 [INSPIRE].

[68] S.K. Raut, R.S. Singh and S.U. Sankar, Magical properties of 2540 KM baseline Superbeam Experiment, Phys. Lett. B 696 (2011) 227 [arXiv:0908.3741] [INSPIRE].

[69] A. Dighe, S. Goswami and S. Ray, 2540 KM: bimagic baseline for neutrino oscillation parameters, Phys. Rev. Lett. 105 (2010) 261802 [arXiv: 1009.1093] [INSPIRE].

[70] S.K. Agarwalla, P. Huber, J. Tang and W. Winter, Optimization of the Neutrino Factory, revisited, JHEP 01 (2011) 120 [arXiv: 1012.1872] [INSPIRE].

[71] S. Prakash, S.K. Raut and S.U. Sankar, Getting the Best Out of T2K and NOvA, Phys. Rev. D 86 (2012) 033012 [arXiv:1201.6485] [InSPIRE].

[72] K. Dick, M. Freund, M. Lindner and A. Romanino, CP violation in neutrino oscillations, Nucl. Phys. B 562 (1999) 29 [hep-ph/9903308] [inSPIRE].

[73] A. Donini, M.B. Gavela, P. Hernández and S. Rigolin, Neutrino mixing and CP-violation, Nucl. Phys. B 574 (2000) 23 [hep-ph/9909254] [INSPIRE]. 